\title{
Jupiter's main auroral oval observed with HST-STIS
}

\author{
D. Grodent, ${ }^{1}$ J. T. Clarke, ${ }^{2}$ J. Kim, ${ }^{2}$ J. H. Waite Jr., ${ }^{3}$ and S. W. H. Cowley ${ }^{4}$ \\ Received 5 March 2003; revised 21 May 2003; accepted 11 June 2003; published 1 November 2003.
}

[1] An extended series of FUV images obtained on 7 days during winter 2000-2001, with fixed pointing, yielded highly accurate tracking of emisson features as Jupiter rotated. They provided newly detailed measurements of the degree of corotation of auroral emissions and their variations with changing central meridian longitude. This 2-month data set provides a statistical average location of the auroral emission and leads to the definition of new "reference ovals." The overall auroral morphology pattern is shown to be fixed in System-III longitude and unchanged over a 5-year period. When arranged in central meridian longitude ranges, the images show a significant contraction of the northern main oval as the central meridian longitude increases from 115 to $255^{\circ}$. The main auroral oval brightness is globally very stable in comparison with its terrestrial counterpart. It is shown to vary with magnetic local time, increasing from noon to dusk and then decreasing again in the magnetic evening. Hectometric emissions observed simultaneously with Galileo and Cassini reveal interplanetary shocks propagating outward from the Sun which may be related to the contraction of the main auroral oval observed in the HST images taken on 14 December 2000. In addition, we find that a brightening and a significant contraction of the main oval observed on 13 January 2001 corresponded to a time of increased solar wind dynamic pressure. INDEX TERMS: 6220 Planetology: Solar System Objects: Jupiter; 2704 Magnetospheric Physics: Auroral phenomena (2407); 2756 Magnetospheric Physics: Planetary magnetospheres (5443, 5737, 6030); 2784 Magnetospheric Physics: Solar wind/magnetosphere interactions; KEYWORDS: Jupiter, aurora, mail oval, planetary magnetospheres, solar wind/magnetosphere interactions

Citation: Grodent, D., J. T. Clarke, J. Kim, J. H. Waite Jr., and S. W. H. Cowley, Jupiter's main auroral oval observed with HSTSTIS, J. Geophys. Res., 108(A11), 1389, doi:10.1029/2003JA009921, 2003.

\section{Introduction}

[2] The Jovian ultraviolet auroras have been extensively studied in the past. They were first observed with the ultraviolet spectrometer (UVS) on board the Voyager 1 and 2 spacecraft [Sandel et al., 1979; Broadfoot et al., 1981]. The main features of the Voyager view of the Jovian auroral morphology were confirmed and greatly expanded by observations with the International Ultraviolet Explorer spacecraft [Clarke et al., 1980; Livengood et al., 1992; Harris et al., 1996]. A new era opened when high-resolution ultraviolet images became available with the Hubble Space Telescope (HST), first with the Faint Object Camera (FOC) [Gérard et al., 1994b; Prangé et al., 1998; Pallier and Prangé, 2001], and later with the Wide Field Planetary Camera (WFPC-2) [Ballester et al., 1996; Clarke et al.,

${ }^{1}$ LPAP, Institut d'Astrophysique et de Géophysique, Université de Liège, Belgium. USA.

${ }^{2}$ Center for Space Physics, Boston University, Boston, Massachusetts,

${ }^{3}$ Space Physics Research Laboratory, University of Michigan, Ann Arbor, Michigan, USA.

${ }^{4}$ Department of Physics and Astronomy, University of Leicester, Leicester, UK.

Copyright 2003 by the American Geophysical Union. 0148-0227/03/2003JA009921
1996; Grodent et al., 1997; Clarke et al., 1998]. These instruments obtained the first high-resolution images of the complex Jovian auroral morphology. A dichotomy has frequently been observed between a narrow single structure arc at System III longitudes $\left(\lambda_{I I I}\right)$ larger than $180^{\circ}$ and multiple arcs and broad diffuse emissions at longitudes less than $180^{\circ}$ in the north. Owing to the limited observing geometry, it was not clear from prior observations if these regions were fixed with respect to Jupiter or represented local time variations within the main oval and polar regions. It was also shown that the equatorial boundary of the emission is located between the 6 Jovian radii $\left(\mathrm{R}_{J}\right)$ and the $30 \mathrm{R}_{J}$ magnetic field line footprints of the VIP4 magnetic model [Connerney et al., 1998]. However, newer observations obtained with the Space Telescope Imaging Spectrograph (STIS) on board HST reveal the location of the footprints of Europa and Ganymede, equatorward of the main oval, which clearly requires the majority of the auroral emission to map to distances greater than $15-20 \mathrm{R}_{J}$ [Clarke et al., 2002a]. The advent of the STIS instrument also revealed new auroral features with an order of magnitude higher sensitivity than earlier cameras. Its higher sensivitity, to $\sim 1$ kiloRayleigh $(\mathrm{kR})$ emissions, allows the tracking of local time features as well as transient features [Waite et al., 2001], giving a much more detailed view of the "diffuse emissions" than was possible with the FOC and WFPC-2 instruments. 
[3] HST images have shown that Jupiter's aurora exhibits three distinct regions based on their locations, the physical regions and processes from which they originate, and their independent variations with time [Clarke et al., 1998]. These three regions appearing in Figure 1 can be summarized as: (1) the satellite footprint emissions, (2) the main oval emissions, and (3) all other emissions poleward of the main oval, which we will refer to as the "polar emissions." The satellite footprint aurora are easily identified by the fact that they remain fixed along magnetic flux tubes connected to Io, Europa, and Ganymede [Clarke et al., 2002a]. The main oval emissions are observed to corotate with Jupiter [Gérard et al., 1994a; Ballester et al., 1996] and are relatively stable, exhibiting variations on time scales of tens of minutes to hours. Imaging studies of the polar emissions have been presented [Pallier and Prangé, 2001] and, by contrast, these emissions vary rapidly, up to the extreme cases represented by the "flares" [Waite et al., 2001; Grodent et al., 2003], which can rise from the background level of a few $k R$ to several megaRayleighs (MR) in brightness in tens of seconds. Three independent papers [Bunce and Cowley, 2001a; Hill, 2001; Southwood and Kivelson, 2001] suggest that the main jovian auroral oval is connected with the magnetosphere-ionosphere coupling current system associated with the breakdown of rigid corotation in the middle magnetosphere region. The main auroral oval may thus result from the upward Birkeland current that enforces partial corotation of plasma moving outward from the Io plasma torus. Measurements of the rotational motion of the low-energy plasma [Hill, 1980; Sands and McNutt, 1988] have shown that the plasma corotates with the planet out to a radial distance of $\sim 20 \mathrm{R}_{J}$. The jovian magnetospheric plasma originates mainly from the moon Io and is injected in near corotation. The iogenic plasma then diffuses slowly outwards, via a process which is thought to be centrifugally driven flux-tube interchange motions. Consequently, as a parcel of plasma moves away from the planet, its angular velocity will tend to fall due to conservation of the angular momentum, though this will partly be offset by the consequent frictional torque imposed at the feet of the field lines due to ionneutral collisions in the ionosphere. Overall, the plasma angular velocity will lag behind corotation, the departure increasing with increasing distance. Measurement of the directional anisotropies of energetic ion distributions by the Galileo Energetic Particles Detector [Krupp et al., 2002] show that the global flow pattern of the magnetospheric plasma is predominantly directed in the corotation direction. The flow is subcorotational in most parts of the jovian magnetotail, with flows as low as $20 \%$ of the rigid corotation velocity. As suggested in the theoretical considerations of Vasyliünas [1983], Galileo observed a pronounced local time asymmetry in the flow pattern [Krupp et al., 2002]. The ions are nearly corotating in the dawn-noon sector, where the plasma is compressed and flows more rapidly, and significantly subcorotating in the noon-dusk sector, where the plasma expands and slows down.

[4] Estimation of the field-aligned currents [Cowley and Bunce, 2001] suggests the existence of field-aligned voltages of the order of $\sim 100 \mathrm{kV}$ responsible for the acceleration of primary auroral electrons to $\sim 100 \mathrm{keV}$, giving rise to an auroral brightness in the main oval on

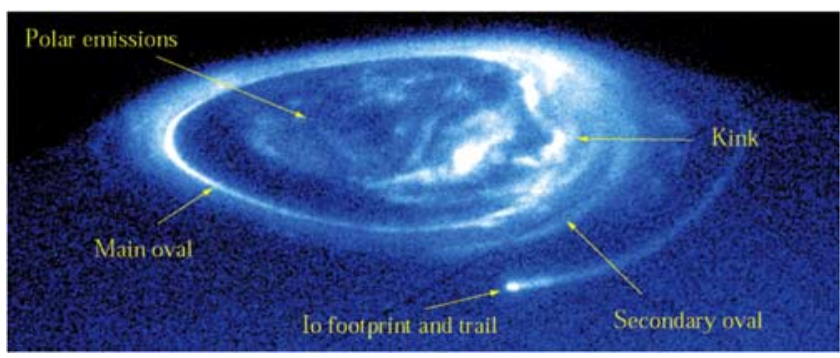

Figure 1. Raw HST-STIS image showing the FUV auroral emission at the north pole of Jupiter. The image was taken on 14 December 2000 through the Clear aperture. Photons were accumulated for $110 \mathrm{~s}$ and the CML at midexposure was $161.6^{\circ}(\mathrm{S} 3)$. Arrows point at the main auroral features: the main and secondary ovals, the Io footprint, and its trailing tail. The polar emissions, including the "flares," appear poleward of the main oval. The distorted part of the main oval (the "kink") is also shown.

the order of a few MR, in agreement with the observations reported here. These authors, as well as Southwood and Kivelson [2001], also suggest that the primary modulation of these auroras by the solar wind will be via the dynamic pressure of the wind, which controls the size of the magnetospheric cavity. When the dynamic pressure of the wind is low, the magnetosphere is expanded, the plasma flows are slow, and the coupling currents and auroral intensity are large. When, on the other hand, the dynamic pressure of the wind is higher, the magnetosphere is compressed, the flows are faster, and the coupling currents and auroral intensity are reduced. Modeling of this process by Cowley and Bunce [2003a, 2003b] indicates that a significant modulation in auroral intensity should be produced. We may similarly suppose that systematic variations of the magnetospheric plasma velocity with local time will also result in related systematic variations in auroral luminosity, in the sense of anticorrelation.

[5] It is well understood that the dynamics of Jupiter's magnetosphere is dominated by the effects of rapid planetary rotation and the massive source of plasma at the moon Io, leading to the formation of high- $\beta$ (i.e., large ratio of plasma pressure to magnetic field pressure) plasma sheet in the middle magnetopshere. By contrast, the physics of the Earth's magnetosphere is dominated by the solar wind interaction and generally by the entry of solar wind plasma at the magnetopause. At the same time, the solar wind does impart both noon-midnight and dawn-dusk asymmetries to Jupiter's magnetosphere (from which others follow), and in this sense there are known to be diurnal variations in the magnetospheric dynamics. The exact dependence of magnetospheric processes and the dependence of the consequent aurora on solar wind and internal processes, however, are not well understood. The scope of this paper is to provide a detailed analysis of Jupiter's main auroral oval observed with HST-STIS and to point out changes in the auroral emission which may be controlled by the solar wind.

\section{Observations and Data Reduction}

[6] This section provides technical information on data acquisition and reduction. It also addresses the problem of 
Table 1. Characteristics of the Seven Data Sets Obtained With HST-STIS (Clear) During the Observation Campaign ${ }^{\text {a }}$

\begin{tabular}{ccccccc}
\hline Date & DOY & Start, UT & End, UT & Num. & Pole & CML Range \\
\hline 14 Dec 00 & 349 & 1046 & 1414 & 19 & $\mathrm{~N}$ & $162-294$ \\
& & 1652 & 1727 & 11 & $\mathrm{~S}$ & $350-23$ \\
16 Dec 00 & 351 & 1100 & 1611 & 21 & $\mathrm{~N}$ & $111-299$ \\
& & 1708 & 1740 & 8 & $\mathrm{~S}$ & $333-355$ \\
18 Dec 00 & \multirow{3}{*}{353} & 1409 & 1621 & 11 & $\mathrm{~N}$ & $167-248$ \\
& & 0927 & 1313 & 19 & $\mathrm{~S}$ & $356-133$ \\
28 Dec 00 & \multirow{2}{*}{363} & 1159 & 1230 & 8 & $\mathrm{~N}$ & $154-176$ \\
& & 0534 & $11: 02$ & 21 & $\mathrm{~S}$ & $281-120$ \\
13 Jan 01 & \multirow{2}{*}{3} & 1650 & 1902 & 11 & $\mathrm{~N}$ & $219-299$ \\
& & 1958 & 2344 & 13 & $\mathrm{~S}$ & $333-109$ \\
20 Jan 01 & \multirow{2}{*}{20} & 1550 & 1806 & 12 & $\mathrm{~N}$ & $156-297$ \\
& & 1241 & 1943 & 11 & $\mathrm{~S}$ & $42-122$ \\
21 Jan 01 & \multirow{2}{*}{21} & 2221 & 2301 & 2 & $\mathrm{~N}$ & $183-207$ \\
& & 1600 & 2124 & 22 & $\mathrm{~S}$ & $312-148$ \\
\hline
\end{tabular}

${ }^{\mathrm{a}}$ The fifth column (Num.) gives the number of images taken at each pole in a data set.

spatial accuracy, which is tied to the fact that only a fraction of the Jovian disk appears in the field of view of the STIS camera at any one time.

\subsection{Observations}

[7] During the winter of 2000-2001, the STIS camera obtained 207 far-ultraviolet (FUV) images of the auroral emission at Jupiter's poles (Figure 1). These observations were completed directly before and after Cassini's closest approach of Jupiter on 30 December 2000. They span a period of 6 weeks (Table 1) starting on 14 December 2000 and ending on 21 January 2001. Table 2 lists the major ephemeris parameters of Jupiter during the observation period.

[8] In this work, we analyzed the 188 images that were taken with the photon-counting detector 25MAMA (MultiAnode Micro channel Array) using the Clear aperture (no filter). In this mode, the solar-blind detector has a bandpass ranging from 115 to $170 \mathrm{~nm}$ and is sensitive to the $\mathrm{H}_{2}$ Lyman and Werner bands as well as the strong H Lyman- $\alpha$ line. Most of the images were taken during dark-time, that is the 45-min period during which HST is in the shadow of the Earth and therefore minimal contamination is expected from the geocoronal Lyman- $\alpha$ emission. There are $1024 \times 1024$ pixels in the MAMA array, their size is $0.02437^{\prime \prime}$, which provides a field of view (FOV) of $24.7^{\prime \prime} \times 24.7^{\prime \prime}$ with a $\sim 0.08^{\prime \prime}$ full width at half maximum point spread function (PSF). For a direct comparison the images were all scaled in pixel size to display Jupiter as it would appear at a distance of 4.2 Astronomical Units (AU). The distance subtended by one pixel on the field of view projected at Jupiter is then $\sim 74 \mathrm{~km}$. At that distance a resolution element corresponds to $\sim 300 \mathrm{~km}$ on the planet.

[9] All the images were accumulated for $\sim 100 \mathrm{~s}$, during which Jupiter rotates by $\sim 1^{\circ}$. This rotation introduced a faint smearing of the images which, for a surface feature located at the central meridian longitude (CML) and at $60^{\circ}$ latitude (a severe case), is on the order of 8 pixels, that is approximately two times the PSF. The blurring is relatively less as one approaches the east or west limb, where the rotational motion is more along the line of sight.

[10] The guiding of the STIS aperture was executed in fine lock mode using onboard acquisition. This method provides a guiding accuracy of $0.007^{\prime \prime}$ ( $\frac{1}{4}$ of a pixel). Apart from one occasion, the guide stars remained the same during each data set (each day). Therefore it is assumed that the pointing did not change between two groups of images taken on the same day (at the same pole). However, it should be pointed out that the Fine Guidance Sensor occasionally loses one of the two guide stars, resulting in a slight roll of the telescope, causing a small offset of the pointing. This entire data set represents a major improvement over previous observations, since the locations of auroral emission regions could be observed "continuously" for several hours.

\subsection{Image Processing}

[11] All auroral images were reduced from the initial data files using programs developed and tested at the University of Michigan, derived from routines which were developed for WFPC-2 images. As part of our data reduction process, the images were flat-fielded using the most recent low-order flat field and pixel-to-pixel files provided by the Space Telescope Science Institute (STScI). Dark counts were subtracted using the algorithm of Landsman [1998] and the appropriate STScI dark files. Geometric distortions were corrected by using the method described by Walsh et al. [2001]. Finally, the size of the images was modified in order to display Jupiter at a standard distance of 4.2 AU. The STIS/FUV-MAMA clear counts were converted in flux using a factor of 0.0013 count per pixel per second for $1 \mathrm{kR}$ of $\mathrm{H}_{2}$ plus Lyman- $\alpha$. This conversion factor was calculated based on a synthetic UV spectrum of $\mathrm{H}_{2}$ [Gérard et al., 2002], including the Lyman- $\alpha$ line (contributing $\sim 15 \%$ of the total $\mathrm{H}_{2}$ spectrum) and assuming a constant methane attenuation of 0.4 below $\sim 130 \mathrm{~nm}$, giving rise to an average FUV color ratio of 2.5 [Gustin et al., 2002]. With the same spectrum, accounting for the pixel area and Jupiter-Earth distance leads to an emitted power of $1.14 \times$ $10^{8} \mathrm{~W} /$ count pixel $^{-1} \mathrm{~s}^{-1}$.

\subsection{Image Projection and Accuracy}

[12] The $25^{\prime \prime} \times 25^{\prime \prime}$ field of view of STIS captures approximately one-fourth of the Jovian disk. As a result, a limb-fitting procedure is necessary to determine the center position of the planet. The procedure consists of fitting the Jovian limb with a model planetary disk. Afterward, each pixel of the initial image is assigned a planetocentric latitude and a $\lambda_{I I I}$ longitude. This procedure allows one to project the initial image on any kind of map, such as a $\lambda_{I I I}$ map on which features fixed on the planet or corotating with the planet always appear at the same location. Conversely, any feature leading or lagging corotation moves from one projected image to the other and can therefore be easily pinpointed. A particular case in the Northern Hemisphere is

Table 2. Major Ephemeris Parameters of Jupiter During the Observation Period

\begin{tabular}{lcc}
\hline \multicolumn{1}{c}{ Parameter } & 14 Dec 2000 1414 UT & 21 Jan 2001 2301 UT \\
\hline Sub-Earth lat. $^{\mathrm{a}}$ & 3.0887 & 2.9296 \\
Phase angle, deg $_{\text {Distance, AU }}$ & 3.6352 & 9.7893 \\
Equ. radius, " & 4.0998 & 4.4984 \\
\hline
\end{tabular}

${ }^{\text {a}}$ Planetocentric latitude in degrees. 


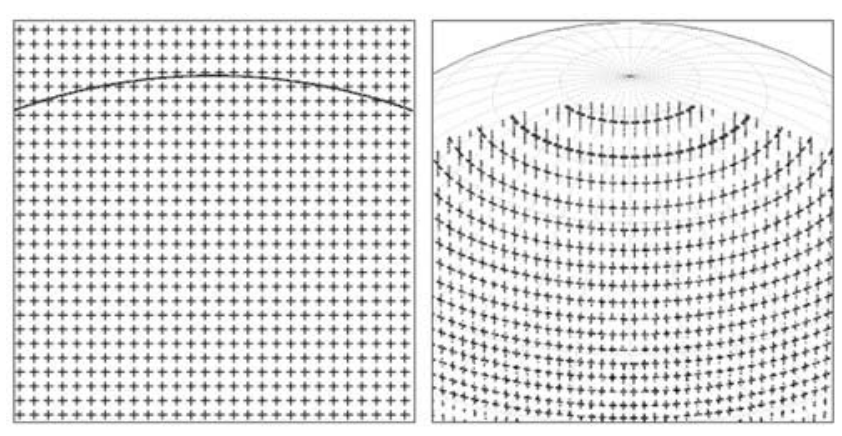

Figure 2. Illustration of the projection method for a particular case. The left panel shows the initial test pattern image; the curve represents the portion of the planetary limb that falls into the FOV of the STIS MAMA detector. The emission has been symbolized by plus symbol marks. The right panel displays the projected view for a line of sight intercepting Jupiter at $30^{\circ}$ latitude. Note the stretching of the plus symbol marks as one nears the limb. On the left panel we discarded the emission appearing above a model limb, located $400 \mathrm{~km}$ below the actual limb.

shown in the left-hand panel of Figure 2, where the STIS image plane has been filled with equidistant plus symbol marks. In the right-hand panel these marks have been projected onto the planet, in a projection where longitude $180^{\circ} \lambda_{I I I}$ is facing the observer and the planet is tilted by $30^{\circ}$ for a better view of the north polar region. It can be seen that there is a growing uncertainty in the projection accuracy as one nears the limb, as seen from the stretching of the plus symbol marks.

[13] A further uncertainty in identifying locations on the planet arises from the error in our determination of the planet center. To quantify how this error affects different regions, an accuracy map showing the error introduced by the limb fitting procedure has been generated. The map for the northern aurora is shown in Figure 3. It was calculated by projecting a synthetic image on an equidistant cylindrical map. The same image was then projected a second time with a shift of the center position of 4 pixels perpendicular to the CML (actually an average case). The distance between each individual point of the two projected images was then measured and recorded on a longitude-latitude map. The polar projection of this accuracy map is shown in Figure 3 for an observation at $\mathrm{CML}=180^{\circ}$. Near the limb, the error on the longitude/latitude is largest, increasing to $5^{\circ}$. It can be reduced to less than $3^{\circ}$ (mainly in longitude) by discarding the emission appearing outside an ellipse $400 \mathrm{~km}$ smaller than the actual projected Jovian ellipsoid. It should be noted that the vertical extent of the auroral curtain has been neglected. Instead, it was assumed that the emission originates from a single surface layer at a fixed altitude of $240 \mathrm{~km}$, in agreement with Galileo visible observations which place the emission peak at an altitude of $245 \pm 30 \mathrm{~km}$ [Vasavada et al., 1999]. It should be noted that the energy degradation model described by Grodent et al. [2001a] shows that the UV and visible emissions (the latter likely originating from continuum emission from triplet states of the $\mathrm{H}_{2}$ molecule) are produced at the same altitude. The vertical distribution and its implications for limb brightening are discussed elsewhere [Grodent et al., 1997; Gérard et al., 1998; Grodent et al., 2001b]. The latitudes and longitudes were computed assuming an oblate spheroid with radii corresponding to the assumed height of the auroral emission $(240 \mathrm{~km})$.

\subsection{Southern Auroral Data}

[14] The viewing geometry (positive sub-Earth latitude) for the southern aurora is less favorable for Earth-based observations of Jupiter's aurora. The proximity of the magnetic south pole to the rotation axis restricts the view of the auroral distribution compared with the northern aurora. Moreover, owing to the subsolar longitude and latitude, most of the southern limb was not illuminated by the Sun and did not provide a sharp limb. Therefore the limb-fitting accuracy in the south is more uncertain. However, there is one remarkable exception: on 18 December 2000 the satellite Europa crossed the field of view (FOV) of STIS and gave an absolute pointing reference in six consecutive images. Owing to the limited size of Europa ( $\sim 40$ pixels in STIS FOV) and its fast transit time, the pointing accuracy is approximately \pm 2 pixels. At least for this day the center position was reliably determined. However, the stretching of the emission near the limb persists and the location of the auroral emission in the south remains less accurate. For this reason, we put the emphasis of this paper on the images taken in the north.

\section{Main Auroral Oval}

[15] The long term distribution of Jupiter's auroral emissions appears to be quite stable. Figure 4 compares the auroral morphology from three epochs. The first set is the

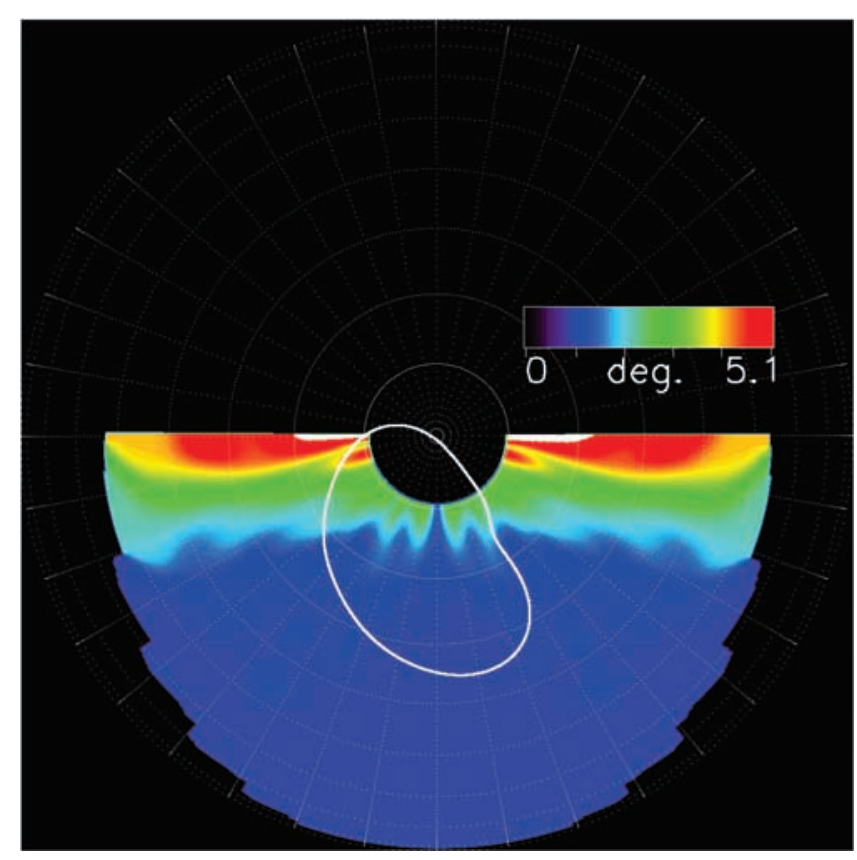

Figure 3. North polar view of the accuracy map. The main auroral oval and a $10^{\circ}$ spaced grid have been overplotted in white. The color table represents the error in degrees (longitude + latitude) due to the accuracy of the limb fitting method. For this map, an error of 4 pixels was assumed in the direction perpendicular to the CML. 

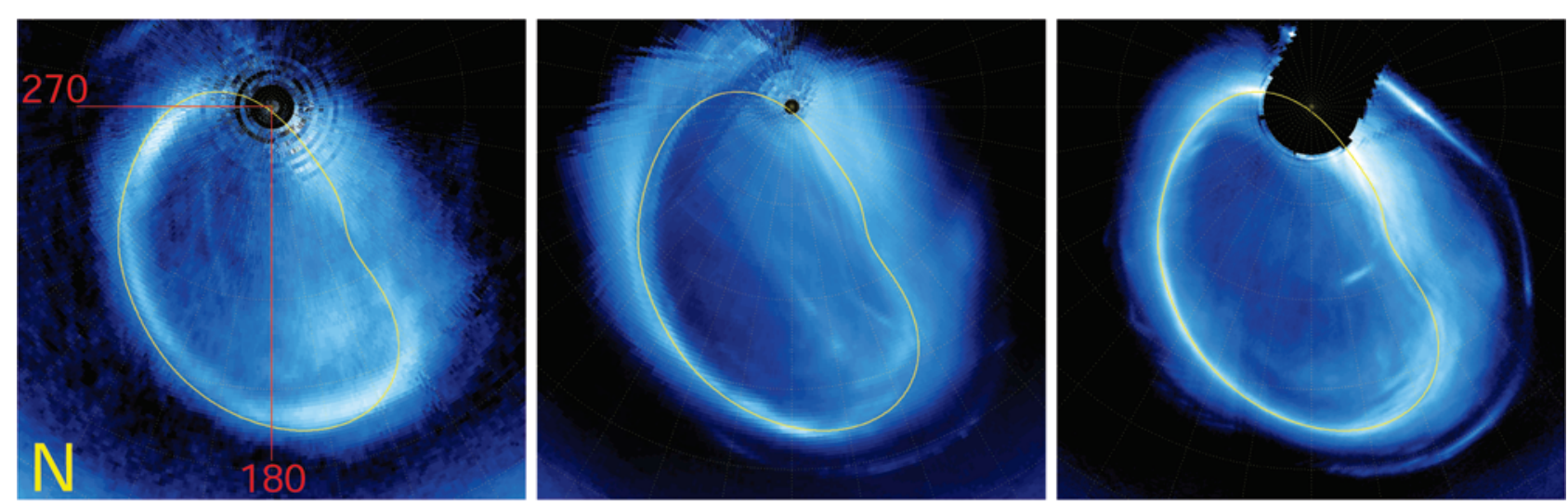

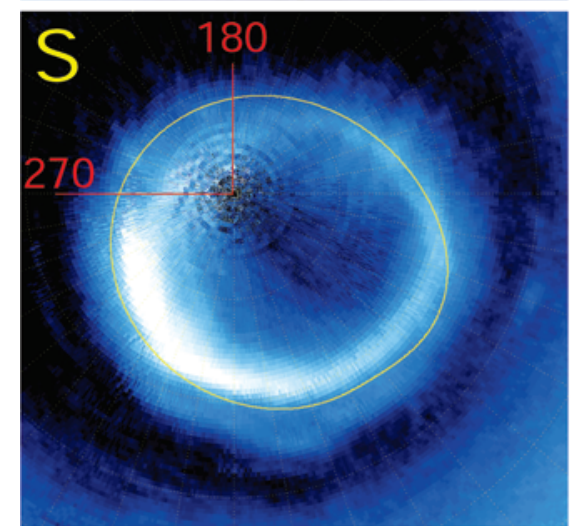

WFPC2 1996
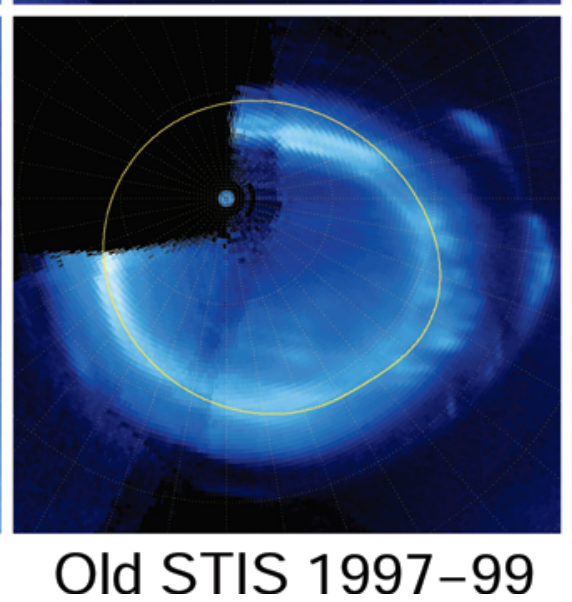

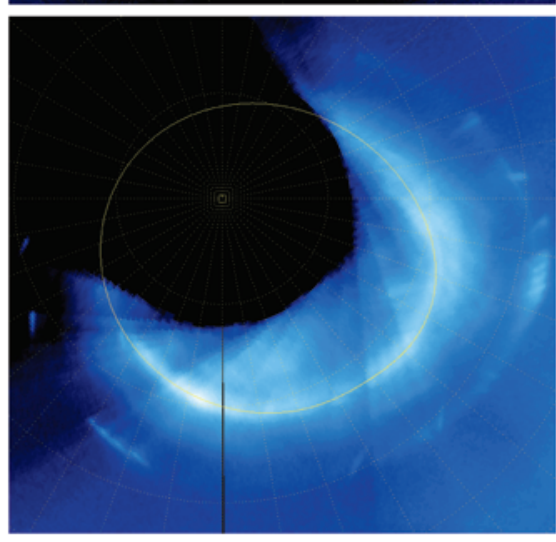

New STIS 2000-01

Figure 4. Long-term comparison of the north (top row) and south (bottom row) auroral emission. The $180^{\circ}$ and $270^{\circ} \lambda_{I I I}$ meridians have been highlighted on a $10^{\circ}$ spaced grid. Left panels show the accumulation of 42 images obtained with the WFPC-2 camera in 1996. Middle panels show the average of 38 images (north) and 18 images (south) taken with the STIS camera between 1997 and 1999. Right panels show the sum of 83 images (north) and 105 images (south) obtained with the STIS camera during the present observation campaign in December 2000 through January 2001. The main reference oval deduced from the new STIS dataset is overplotted on each panel for comparison.

statistical average of 42 images taken with the WFPC-2 camera in 1996. The second set represents the accumulation of 38 images in the north and 18 images in the south, taken sparsely with the STIS camera during a 3-year period from 1997 to 1999. The last set is the present data set and contains 83 images in the north and 105 in the south, obtained during this 2-month HST campaign. As will be discussed below, it is possible to characterize the complex morphology with one single contour that we call "the main reference oval." Overplotting the reference oval deduced from the lastest data set on the three average images allows a comparison of the general morphology. It is clearly shown by the continuous white lines in Figure 4 that the morphology remained stable, even over a 5-year period. The better quality of the new STIS statistical image compared with the old STIS image is mainly due to the improvement of the limb fitting procedure, owing to the stability of the pointing from one image to another. It should be stressed that this improvement was possible only because during the present campaign all the images were taken close in time and on the same guide stars, which requires several consecutive HST orbits on the same day. Note that for the WFPC2 polar projections the peak emission altitude was set to $400 \mathrm{~km}$ [Clarke et al., 1998], that is $160 \mathrm{~km}$ higher than in the STIS data sets. This difference partly explains the location of the WFPC2 main oval poleward of the STIS reference oval (mainly in the south). In any case the difference is on the same order as the uncertainty stemming from the projection and center-finding procedures.

\subsection{Reference Ovals}

[16] We have produced "reference ovals" for various emission regions to aid in the comparison of images taken on different days, with different observing geometries, and with different instruments. Note that like the WFPC-2 reference ovals, these are not strictly statistical ovals but reference locations for the comparison of images. The projection procedure, described in section 2.3, allows a direct comparison of the different images on a single reference frame fixed on the surface of the planet, that is a $\lambda_{I I I}$ map. Figure 5 shows a $\lambda_{I I I}$ polar projection of the sum of the images taken during the observation campaign in the north and in the south, respectively. A total of 83 images were coadded in the north and 105 images in the south. The colocation of the auroral emission on these $\lambda_{I I I}$ polar plots, as well as on the long-term comparison, demonstrates that the bulk of the emission is corotating with the planet. However, it will be shown that individual features appearing 


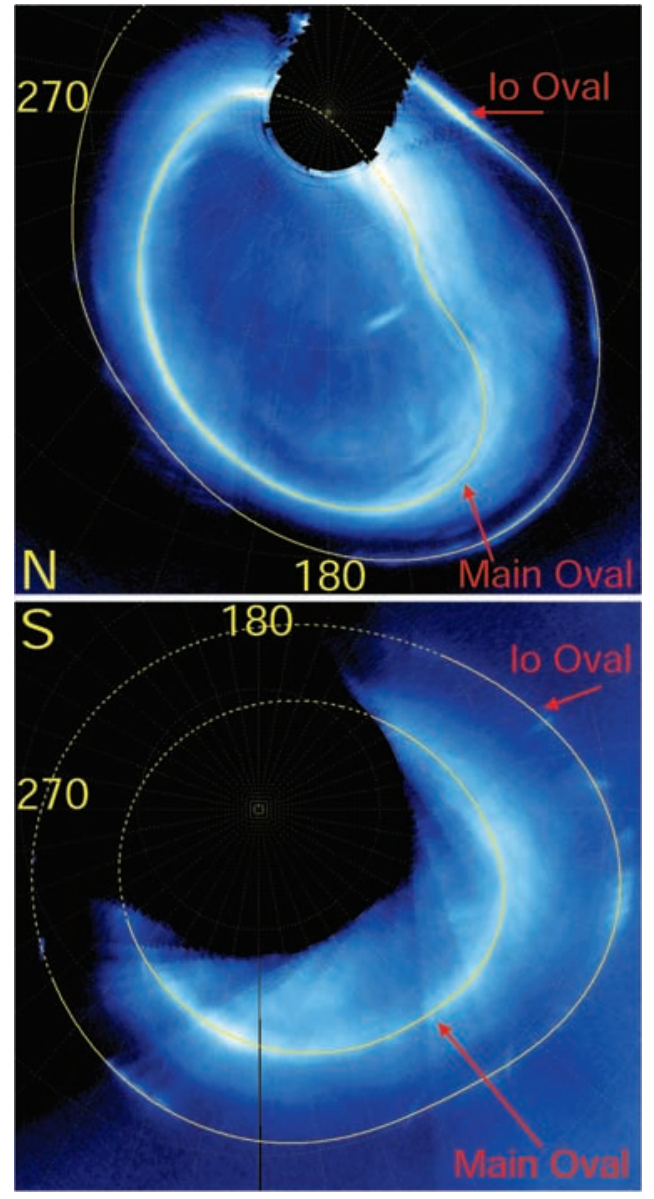

Figure 5. Polar projection $\left(\lambda_{I I I}\right)$ of the reference auroral ovals in the north (upper panel) and in the south (lower panel). The main and Io reference ovals were determined from the sum of the images (shown in blue) obtained during the Cassini Jupiter flyby campaign in December 2000 through January 2001. A $10^{\circ}$ spaced grid with longitude $270^{\circ}$ towards the left is overplotted. In the north, the portion of the Io oval where $290^{\circ}<\lambda_{I I I}<70^{\circ}$ and the portion of the main oval where the latitude exceeds $80^{\circ}$ (dashed lines) are poorly or not constrained by the observations and should be considered as speculative. In the south, the same restriction applies to both ovals where $130^{\circ}<\lambda_{I I I}<310^{\circ}$.

on this pattern are not in corotation. From this statistical average, two "reference ovals" arise which summarize the auroral morphology: (1) the main oval, running through the alignment of the brightest emission, and (2) the Io oval, intercepting the footprint of Io and its trailing tail. These ovals were constructed from several points selected by eye in the brightest portions of the average aurora. In the follwowing sections we will describe the morphology of the main oval and discuss its deviation from rigid corotation.

\subsubsection{Description of the Main Oval}

[17] The main oval connects emission features that may very well map to different regions of the magnetosphere. Therefore it should not be assumed to be a "fixed radial distance" footprint. In the north, at longitudes greater than $180^{\circ}$ the emission along the main oval is almost always very narrow $(\sim 1000 \mathrm{~km})$ and aligned along the reference oval, while at smaller longitudes the emission broadens and tends to break from the main oval (Figure 1). It has been recently proposed [Cowley and Bunce, 2001; Hill, 2001; Southwood and Kivelson, 2001] that the main auroral oval may be interpreted as the ionospheric footprint of the upward Birkeland current (downward electron beam) that enforces partial corotation of magnetospheric plasma moving outward from the Io plasma torus to the outer magnetosphere. According to these models, the equatorial source of these outward field-aligned currents is broadly distributed within the middle magnetosphere current sheet, between inner distances of $\sim 20 \mathrm{R}_{J}$ and outer distances of several tens of $\mathrm{R}_{J}$, bounded by the radial extent of the current sheet. Such distributions are consistent with the mapping deduced from STIS observations of the Europa and Ganymede footprints [Clarke et al., 2002a] which indicate that the bulk of the main oval emission maps outside of Ganymede's orbit at $15 R_{J}$. Cowley and Bunce [2001] developed a simple empirical model of the field and flow in the middle magnetosphere to estimate the field-aligned currents flowing into and out of the equatorial current sheet associated with the breakdown of corotation. They obtain results for three different empirical radial distributions of the angular velocity (equation (17)) characterized by a power index $\mathrm{n}=$ $2,4,6$. As $n$ increases, so does the sharpness of the decrease in angular velocity. With this index, the predicted latitudinal extent of the main oval emission (FWHM) is $\sim 940, \sim 600$, and $\sim 410 \mathrm{~km}$, respectively. After correction for smearing, the observed FWHM of the Gaussian fit of the narrowest part of the main oval (around longitude $220^{\circ}$ ) varies between $0.5^{\circ}(\sim 600 \mathrm{~km})$ and $1.5^{\circ}(\sim 1800 \mathrm{~km})$. Therefore constraining Cowley and Bunce's empirical model with our observations would favor the $\mathrm{n}=2$ parameter. Subsequent work has indeed shown that this model is close to that determined from self-consistent calculations [Cowley et al., 2002]. For this value their model predicts field-aligned acceleration voltages in the range $\sim 50-250 \mathrm{kV}$ giving rise to UV auroral brightness on the order of $\sim 1 \mathrm{MR}$. This value is confirmed by the brightness observed in the main oval which is shown to vary from $\sim 50$ to $\sim 500 \mathrm{kR}$ (it should be noted that the brightness depends on the conversion factor from counts to $\mathrm{kR}$ discussed in section 2.2). If one assumes a $1^{\circ}$-wide arc of the oval in the $170^{\circ}-270^{\circ} \lambda_{I I I}$ longitude portion (the "left ansa"), which maps to the morning-noon sector for CML around $200^{\circ}$, then one obtains a total input power of 0.2 to $2 \times 10^{12} \mathrm{~W}$ in that portion of the main oval (assuming that an energy input flux of $1 \mathrm{~mW} \mathrm{~m}^{-2}$ is necessary to produce $10 \mathrm{kR}$ of $\mathrm{H}_{2}$ emission [Grodent et al., 2001a]), or 0.2 to $2 \times 10^{10} \mathrm{~W}$ per degree of longitude. In the wider regions of the main oval, where the width is up to $3^{\circ}$, the input power is proportionally increased from 0.6 to $6 \times 10^{10} \mathrm{~W}$ per degree of longitude.

[18] A winding emission pattern is observed in the Northern Hemisphere in the $110-150^{\circ} \lambda_{I I I}$ region. We will refer to it as the "kink" (following the suggestion of Ballester et al. [1996]). It was already apparent in FOC images (Pallier and Prangé [2001]) and in WFPC-2 images (first panel of Figure 4) [Ballester et al., 1996; Clarke et al., 1998]. Its brightness distribution is usually extremely variable (it will be shown later that some auroral features in this region lag behind corotation). Despite its high variability, the kink is always present and its bulk structure is both repeatable and in clear corotation, i.e., fixed with respect to 


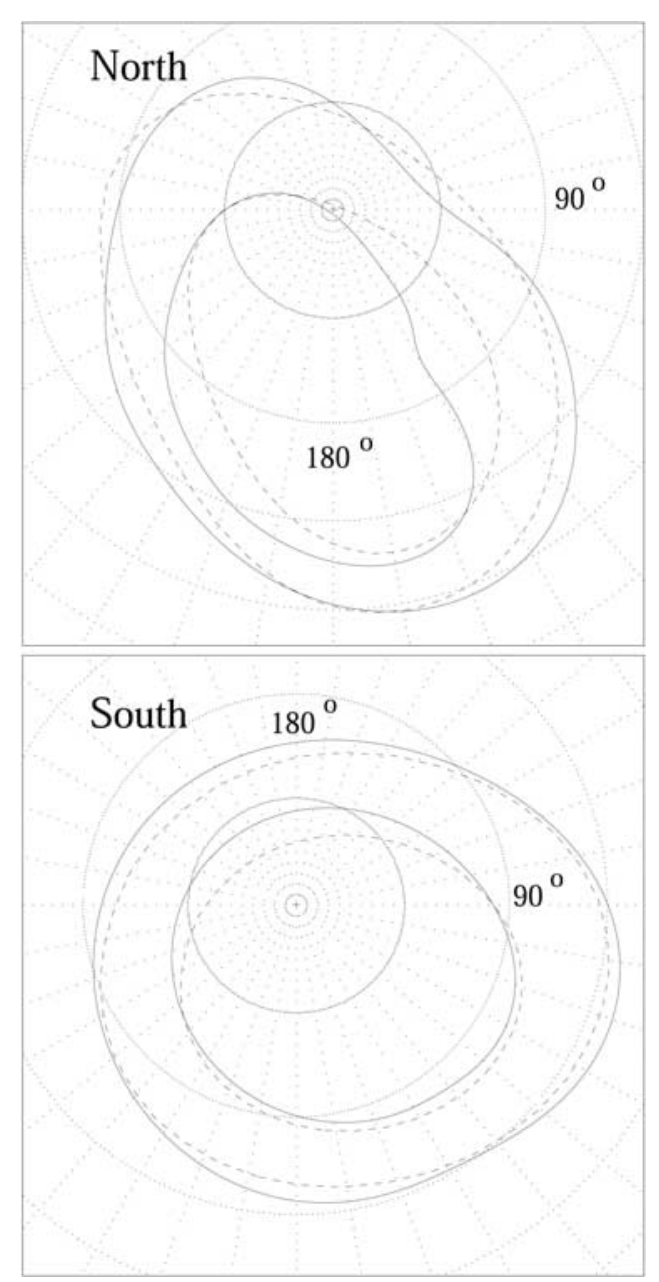

Figure 6. Comparison of the Io and main reference ovals (solid lines) with the VIP4 $5.9 \mathrm{R}_{J}$ (Io) and $30 \mathrm{R}_{J}$ ovals (dashed lines). The Io ovals appear equatorward. The upper panel shows the northern hemisphere and the lower panel displays the southern hemisphere. A $10^{\circ}$ spaced grid is overplotted, and the $90^{\circ}$ and $180^{\circ} \mathrm{S} 3$ longitudes are marked up. In the north, the portion of the Io oval where $290^{\circ}<\lambda_{I I I}<70^{\circ}$ and the portion of the main oval where the latitude exceeds $80^{\circ}$ are poorly or not constrained by the observations and should be considered as speculative. In the south, the same restriction applies to both ovals where $130^{\circ}<\lambda_{I I I}<310^{\circ}$.

Jupiter's magnetic field. A comparison with visible images taken by Galileo [Vasavada et al., 1999] indicates that the kink is also observed in the nightside (see the discussion by Pallier and Prangé [2001]). Figure 6 compares the reference ovals in the north and in the south with the footprints of the magnetic field lines mapping to $5.9 \mathrm{R}_{J}$ (the orbit of Io) and $30 \mathrm{R}_{J}$ given by the VIP4 model [Connerney et al., 1998]. Although the observed and VIP4 ovals are in reasonable agreement (especially in the south), localized deviations occur. In the northern region constrained by STIS images $\left(290^{\circ}>\lambda_{I I I}>70^{\circ}\right)$, the difference between the main oval and the $30 \mathrm{R}_{J}$ oval maximizes in the kink sector (along the $150^{\circ}$ meridian). Various possibilities have been proposed to explain this disturbed structure [Ballester et al.,
1996; Pallier and Prangé, 2001]. Most recently, it has been specifically suggested that this deviation is caused by an internal magnetic anomaly that would locally deform the surface magnetic field [Clarke et al., 2002b; Dessler and Vasyliūnas, 1979]. However, it should be noted that the identification of the main oval emission in the kink with the region driven by upward currents from corotation breakdown is uncertain and other phenomena may as well contribute to the deviation. Another source of deviation stems from the fact that the main oval does not map to $30 \mathrm{R}_{J}$ but to equatorial regions ranging from $15 \mathrm{R}_{J}$ to several tens of $\mathrm{R}_{J}$.

[19] Emissions roughly half as bright as the main oval appear at times equatorward of the main oval. (These are more speculative for the south and therefore we will only address the north). They are best seen in individual images (as in Figure 1) rather than in the average image (upper panel of Figure 5), where they become confused with the background auroral emissions. When the CML ranges from 110 to $220^{\circ}$, the "secondary oval" almost always appears for $100^{\circ}<\lambda_{I I I}<180^{\circ}$. In this region, which corresponds to the kink sector, it follows the same winding path as the main oval. It is much fainter, and most of the time hardly detected, in the $180-210^{\circ}$ region where it gets closer to the main oval and presumably merges with it (around $205^{\circ}$ ). At larger longitudes, it separates from the main oval and is best seen with the dawn limb brightening. It is emphasized that outside the kink sector, the secondary oval is rather speculative. Further analysis is required to confirm its occurrence all along the main oval. The origin of the secondary oval is presently unknown. Stallard et al. [2001], discussing banded auroral emissions observed poleward of the main oval in their own data and those of Pallier and Prangé [2001] and Clarke et al. [1995], suggested that they are produced by a stepwise breakdown in corotation of the equatorial plasma. One possibility is that a similar process may be operative here in forming the secondary oval equatorward of the main oval. This would imply that the auroral emission associated with the Birkeland currents transmitting the torque from the ionosphere to the magnetosphere occur from the Io oval right to the polar region. The secondary oval may then represent a first step, at lower latitudes and lower intensities, of the process of corotation enforcement. It should be noted, however, that the stepwise departure from rigid corotation is purely empirical, while the existing theoretical calculations, such as those by Hill [2001], Cowley et al. [2002], and Nichols and Cowley [2003], all predict a smooth monotonic decline in plasma angular velocity with increasing latitude in the auroral ionosphere.

\subsubsection{Io Oval}

[20] The Io oval connects the footprint of Io and its trailing tail. It is assumed that Io and its tail are on the same oval, though a drift of the tail is possible (the physical origin of the tail is still under debate: see Clarke et al. [2002a], Gérard et al. [2002], and Hill and Vasyliūnas [2002]). It should be noted that we only considered the portion of the trailing tail for which the signal to noise ratio is sufficient to accurately determine its location (that is, up to $30^{\circ}$ tailward, depending on the viewing geometry). Figure 6 shows that in the Northern Hemisphere, the Io oval is likely influenced by the magnetic anomaly described 
Table 3. Polar Coordinates of the Reference Ovals ${ }^{\mathrm{a}}$

\begin{tabular}{|c|c|c|c|c|}
\hline$\lambda_{I I I}$ longitude & North Main & Latitude Io & South Main & Latitude Io \\
\hline 20. & - & 81.89 & -68.28 & -60.59 \\
\hline 30. & - & 82.33 & -67.80 & -60.36 \\
\hline 40. & - & 82.61 & -67.40 & -59.58 \\
\hline 50. & - & 82.66 & -66.95 & -58.37 \\
\hline 60. & - & 82.50 & -67.08 & -57.43 \\
\hline 70. & - & 82.07 & -68.04 & -57.30 \\
\hline 80. & - & 81.25 & -69.66 & -58.16 \\
\hline 90. & - & 79.57 & -71.58 & -59.99 \\
\hline 100 & - & 75.89 & -73.48 & -62.53 \\
\hline 110. & - & 70.14 & -75.17 & -65.24 \\
\hline 120. & - & 64.82 & -76.67 & -67.75 \\
\hline 130. & - & 59.45 & -77.93 & -69.85 \\
\hline 140. & 84.94 & 54.26 & -78.92 & -71.48 \\
\hline 150. & 73.46 & 50.48 & -79.77 & -72.70 \\
\hline 160. & 56.32 & 48.77 & -80.41 & -73.63 \\
\hline 170. & 54.65 & 48.94 & -80.90 & -74.31 \\
\hline 180. & 55.43 & 50.49 & -81.27 & -74.78 \\
\hline 190. & 57.44 & 52.97 & -81.49 & -75.04 \\
\hline 200 & 60.11 & 55.94 & -81.61 & -75.13 \\
\hline 210. & 63.19 & 58.77 & -81.61 & -75.06 \\
\hline 220 & 66.21 & 61.00 & -81.51 & -74.85 \\
\hline 230. & 69.24 & 62.98 & -81.30 & -74.49 \\
\hline 240. & 72.47 & 65.08 & -80.98 & -74.00 \\
\hline 250 & 75.27 & 67.02 & -80.57 & -73.40 \\
\hline 260. & 77.66 & 68.56 & -80.02 & -72.65 \\
\hline 270. & 80.00 & 69.68 & -79.36 & -71.80 \\
\hline 280. & 82.64 & 70.53 & -78.62 & -70.82 \\
\hline 290. & 86.78 & 71.32 & -77.73 & -69.77 \\
\hline 300. & - & 72.14 & -76.78 & -68.59 \\
\hline 310. & - & 73.11 & -75.72 & -67.34 \\
\hline 320. & - & 74.36 & -74.59 & -66.03 \\
\hline 330. & - & 75.84 & -73.46 & -64.70 \\
\hline 340. & - & 77.40 & -72.28 & -63.41 \\
\hline 350. & - & 78.91 & -71.11 & -62.26 \\
\hline 360. & - & 80.11 & -70.04 & -61.37 \\
\hline
\end{tabular}

${ }^{\text {a }}$ The first column is the $\lambda_{I U}$ longitude in degrees, followed by the corresponding planetocentric latitude (degrees) for the main and Io reference ovals. In the north, the portion of the Io oval where $290^{\circ}<\lambda_{I I I}$ $<70^{\circ}$ and the portion of the main oval where the latitude exceeds $80^{\circ}$ are poorly or not constrained by the observations and should be considered as speculative. In the south, the same restriction applies to both ovals where $130^{\circ}<\lambda_{I I I}<310^{\circ}$.

above (kink in the main oval) and forms an equatorward deviation around $\lambda_{I I I}=90^{\circ}$. From the morphology of the kink in the ovals one would define a deviation central point at latitude $75^{\circ}$ and $125^{\circ} \lambda_{I I I}$ that might relate to the center of a magnetic anomaly. Since the Io oval maps to a short distance from Jupiter $\left(6 \mathrm{R}_{J}\right)$, where very few external perturbations to the internal magnetic field are expected, this feature has been used to provide a consistent mapping for the entire HST campaign data set (Table 1) investigated here. The limb-fitting procedure was iteratively run until the traces of Io fell on one single oval for all data sets.

\subsubsection{Coordinates of the Reference Ovals}

[21] The reference ovals in the north and in the south were fit by a partial sum of sine and cosine series in a shifted coordinate system approximately centered on the middle of the ovals. The series was based upon longitude and latitude coordinates in order to generate a smooth and closed loop around the pole. In determining the coefficients of the series, various numbers of coefficients were tried in order to fit the sampled points in the observed images using the singular value decomposition method. We assumed that the uncertainty on the determination of the latitude, for a given longitude, is $5 \%$, which gave rise to a minimized $\chi^{2}$ less than 0.01 . The resulting planetocentric latitude and $\lambda_{I I I}$ longitude values are listed in Table 3 and displayed in Figure 5. In the north, the portion of the Io oval where $290^{\circ}<\lambda_{I I I}<70^{\circ}$ and the portion of the main oval where the latitude exceeds $80^{\circ}$ are poorly or not constrained by the observations and should be considered as speculative. In the south, the same restriction applies to both ovals where $130^{\circ}<\lambda_{I I I}<310^{\circ}$.

\subsection{Overall Brightness Variations}

[22] The main oval usually contributes $\sim 75 \%$ of the total auroral brightness, the rest being due to the emission appearing inside the main oval (the polar emission). The brightenings that are often observed in the polar emission (the flares) only slightly modify this ratio, though exceptional events such as the one described by Waite et al. [2001] contribute more significantly to the total emission. Accordingly, one may consider that the total auroral brightness is predominently controlled by the main oval aurora. The total brightness as a function of CML and time is plotted in Figure 7 for the north aurora. In the top panel of Figure 7 the brightness was measured from the raw images corrected for background emission only.

[23] In order to compare the total brightnesses for different days (bottom panel of Figure 7), the variations owing to the viewing geometry, mainly depending on the longitude facing the observer (the CML), have to be considered. Accordingly, following the method used by prior observers [Clarke et al., 1980; Skinner et al., 1984; Livengood et al., 1992; Gladstone et al., 2002], the geometric factor was assumed to be defined by the cross-sectional area of the polar region, as seen by the observer, limited by the main auroral oval. The counts in each image were divided by this area to correct for the observing geometry. The emission occurring outside the planetary disk was rejected because it is strongly CML dependent and because it cannot be accounted for by the correction for visible surface area. Since these corrections are approximate, a smooth systematic variation of the brightness as a function of the CML remains for each day. It was attenuated by applying a thirdorder polynomial baseline correction, the same for each day. Since the viewing geometry effects are smooth in nature (the FWHM of the quasi-Gaussian curve representing the correction for visible surface as a function of the CML is on the order of $140^{\circ}$, that is, a 4-hour rotation), it may be asserted that the remaining fluctuations around the daily average displayed on the bottom panel of Figure 7 stem from intrinsic brightness variations. It should be noted however that the importance of these intrinsic variations may themselves be driven by CML dependent processes.

[24] Jupiter's aurora are far more stable than the Earth's. The auroral emissions are always present, and when the total emissions are compared, they change little from day to day. The average uncorrected and corrected light curves do not allow one to highlight any definite trend in this 2-month data set. If one assumes, as a rule of thumb, that an energy input flux of $1 \mathrm{~mW} \mathrm{~m}^{-2}$ is necessary to produce $10 \mathrm{kR}$ of $\mathrm{H}_{2}$ emission [Grodent et al., 2001a], then the total power injected in the northern auroral atmosphere is on the order of $\sim 10^{13} \mathrm{~W}$. The overall brightness of the northern aurora is steady with typically $20 \%$ variations from day to day, as seen in Figure 7. The one exception was 13 January 2001, 


\section{North aurora}
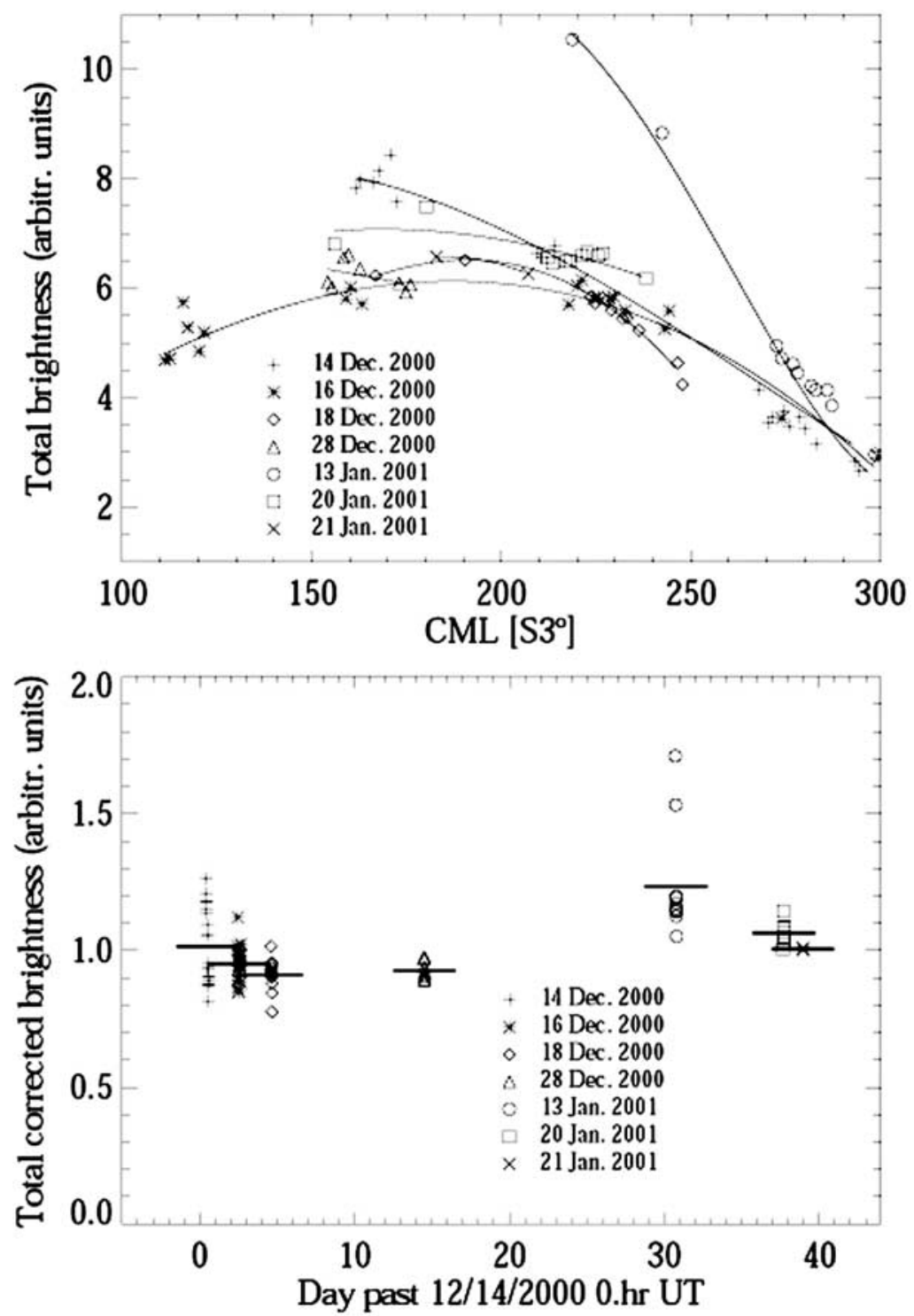

Figure 7. Total auroral brightness (in arbitrary unit) for the north aurora as a function of CML (top panel) and as a function of time (bottom panel). The brightnesses in the top panel have been corrected for background emission, and polynomial interpolation has been overplotted to facilitate the reading. The brightnesses in the bottom panel have been corrected for background emission and for the viewing geometry (visible portion of the aurora and emission appearing above the planetary disc, see text). Different symbols have been used to discriminate the seven observation days. The horizontal bars in the bottom panel represent the average brightness for a particular day. The lowest brightness was observed on 18 December 2000 and the brightest aurora occurred on 13 January 2001. The error on the total auroral brightness is $\sim 15 \%$, less than the daily scatter of datapoints in the bottom panel.

when the total emissions were 1.5-2 times brighter than on other days. This increase by nearly a factor of two is reminiscent of the observations of the total auroral emission by the Cassini UVIS [Gurnett et al., 2002], in which the auroral brightness varied by a similarly small fraction from day to day but increased at one epoch by a factor of $2-3$ in late November 2001. Cassini measurements of the solar wind pressure at Jupiter indicated an increase in dynamic pressure and expected compression of the magnetosphere at the time of this event [Gurnett et al., 2002]. At the same time, an increase in the hectometric radio emissions indicated stronger auroral activity coincident with the UV brightness increase (see discussion in section 3.5). Unfortunately, there is no information on which of the three Jovian auroral regions was responsible for this increase. In the present data set, we can state that the entire main oval has occasionally undergone brightenings (as was the case on 13 January 2001) and the overall polar emission remained 


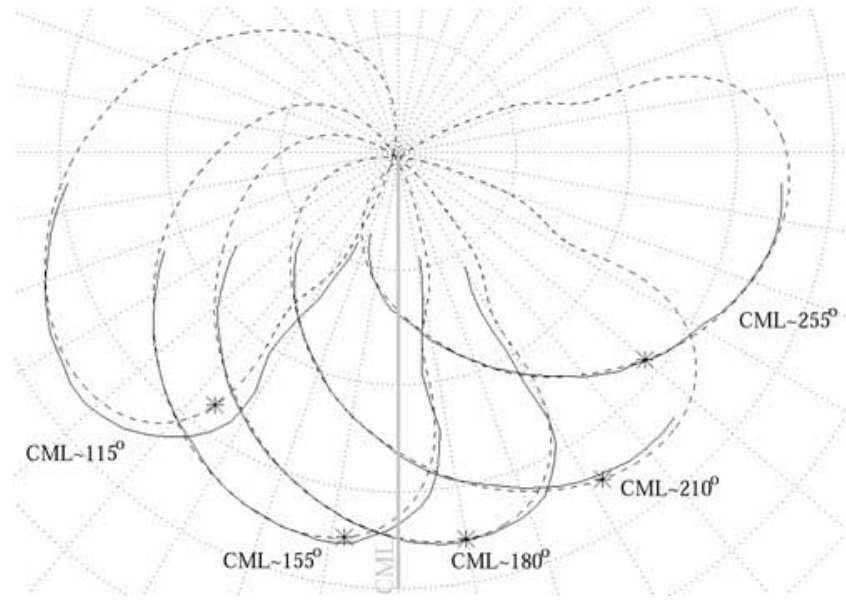

Figure 8. North polar projection in a reference frame fixed relative to the Sun (the Sun is directed towards the bottom of the page and the CML is highlighted) showing the contraction of the main oval emission with respect to the reference oval (dashed lines). Five CML ranges centered on $115^{\circ}, 155^{\circ}, 180^{\circ}, 210^{\circ}$, and $255^{\circ}$ were selected in the north images obtained during the present campaign (they were chosen for the visibility of the main oval). For each range an oval of reference (continuous line) was determined from the brightest average emission of the main oval. The plot shows the contraction of the main oval as the CML increases from 115 to $255^{\circ}$. A $10^{\circ}$ spaced grid is overplotted. The stars indicate the ionospheric footprints of the magnetic field lines which intercept, for each CML, the equatorial plane at noon. In other words, the stars mark noon in MLT as determined from the VIP4 magnetic model at $25 \mathrm{R}_{J}$.

relatively stable in time, apart from 14 December 2000 when a flaring activity was detected.

[25] The uncertainty from photon counting (Poisson statistics) is less than $1 \%$ for the bulk of the northern auroral emission in $\sim 100 \mathrm{~s}$. However, the FUV spectrum in the STIS MAMA clear band pass is variable and the sensitivity of the detector as a function of the wavelength is nonlinear and time dependent. Accordingly, an accumulated absolute error of $15 \%$ has been considered. The light curve as a function of time (bottom panel of Figure 7) is clearer, though tied to the accuracy of the corrections, and allows one to trace a slight trend through the horizontal bars (the bars represent the average brightness during each day). It would place the 18 December 2000 data set at the lowest brightness activity and the 13 January 2001 at the highest activity.

\subsection{Deviation of the Main Oval From Rigid Corotation}

[26] The stable pointing and tracking during the campaign makes it possible to measure motions and changes in brightness of specific auroral emission features as they move with respect to the System III (S3) longitude system. It is also possible to divide the main oval into longitudinal sections, and to determine any changes in latitude and in brightness of these subsections with local time. These measurements directly test the theoretical predictions [Cowley and Bunce, 2001, 2003a, 2003b; Southwood and Kivelson, 2001; Waite et al., 2001; Khurana, 2001] of the changes in auroral activity which are expected to accompany changes in the angular velocity of the equatorial plasma. These studies are presented in this section, but before further discussion it is important to state the difference between local time (LT) and magnetic local time (MLT). A feature fixed in LT is fixed relative to the subsolar position, while a feature fixed in MLT implies that it is the magnetospheric region to which this feature is mapping that is fixed relative to the Sun. An MLT behavior is much more complex than an LT behavior. As an example, a typical MLT motion is that of an auroral feature sliding along the main oval while the planet (and the main oval) is rotating. The complexity (in the north) stems from the tilt of the magnetic axis and from the distorted shape of the magnetic field, especially the azimuthal component. Indeed, the VIP4 model, which has been used in this study, shows that at the orbit of Ganymede $\left(15 \mathrm{R}_{J}\right)$, the difference between the surface longitude of a given magnetic field line and the correpsonding longitude at the magnetic equator can be up to $80^{\circ}$. Figure 8 illustrates this difference. In this figure, the stars indicate the ionospheric footprint of the magnetic field lines on the main oval which intercept, for a given CML, the equatorial plane at noon. In other words, the stars mark noon in MLT. It readily appears that magnetic local noon is different from local noon which is roughly defined by the CML (rigorously speaking, this is the case when the subsolar and sub-Earth longitudes are equal). At this point it is important to mention that the VIP4 model becomes increasingly uncertain when one moves to higher latitude but, in principle, it is appropriate for the main oval mapping to $20-25 R_{J}$.

\subsubsection{Changes in Morphology}

[27] After all the images have been projected consistently for each data set, it is possible to arrange them according to their CML instead of their time. By comparing the emission in a given longitudinal range, observed at different CMLs, it is possible to discriminate the emission features corotating with the planet from the features whose position and/or morphology varies with CML. Table 4 describes the eight CML ranges that were defined for the northern images, designed to contain at least six images.

[28] The first morphological change that we report is illustrated in Figure 8. It takes the form of a contraction of the main oval, compared with the position of the average reference oval, as the CML increases from 115 to $255^{\circ}$. Though relatively small $\left(\sim 2^{\circ} \pm 0.8^{\circ}\right.$ in latitude), this motion is also measured in consecutive raw images, obtained with the same guide stars during a single day. Therefore it is unlikely to be an artifact introduced by the data reduction.

Table 4. Number of Images Taken in the Different CML Ranges ${ }^{\mathrm{a}}$

\begin{tabular}{ccc}
\hline CML Range $^{\circ} \lambda_{I I I}$ & Num. & Dates \\
\hline $110-120$ & 6 & $16 \mathrm{~d}$ \\
$155-160$ & 7 & $16 \mathrm{~d}, 28 \mathrm{~d}, 20 \mathrm{j}$ \\
$160-170$ & 9 & $14 \mathrm{~d}, 16 \mathrm{~d}, 18 \mathrm{~d}, 28 \mathrm{~d}$ \\
$170-190$ & 6 & $18 \mathrm{~d}, 28 \mathrm{~d}, 20 \mathrm{j}, 21 \mathrm{j}$ \\
$205-220$ & 10 & $14 \mathrm{~d}, 16 \mathrm{~d}, 13 \mathrm{j}, 20 \mathrm{j}, 21 \mathrm{j}$ \\
$220-240$ & 20 & $16 \mathrm{~d}, 18 \mathrm{~d}, 20 \mathrm{j}$ \\
$240-270$ & 6 & $14 \mathrm{~d}, 16 \mathrm{~d}, 18 \mathrm{~d}, 13 \mathrm{j}$ \\
$270-295$ & 17 & $14 \mathrm{~d}, 13 \mathrm{j}$ \\
\hline
\end{tabular}

${ }^{\mathrm{a}}$ The dates column lists the day(s) represented in a range (two first digits), $\mathrm{d}$ for December 2000 and $\mathrm{j}$ for January 2001. Only the data in the north are considered. 


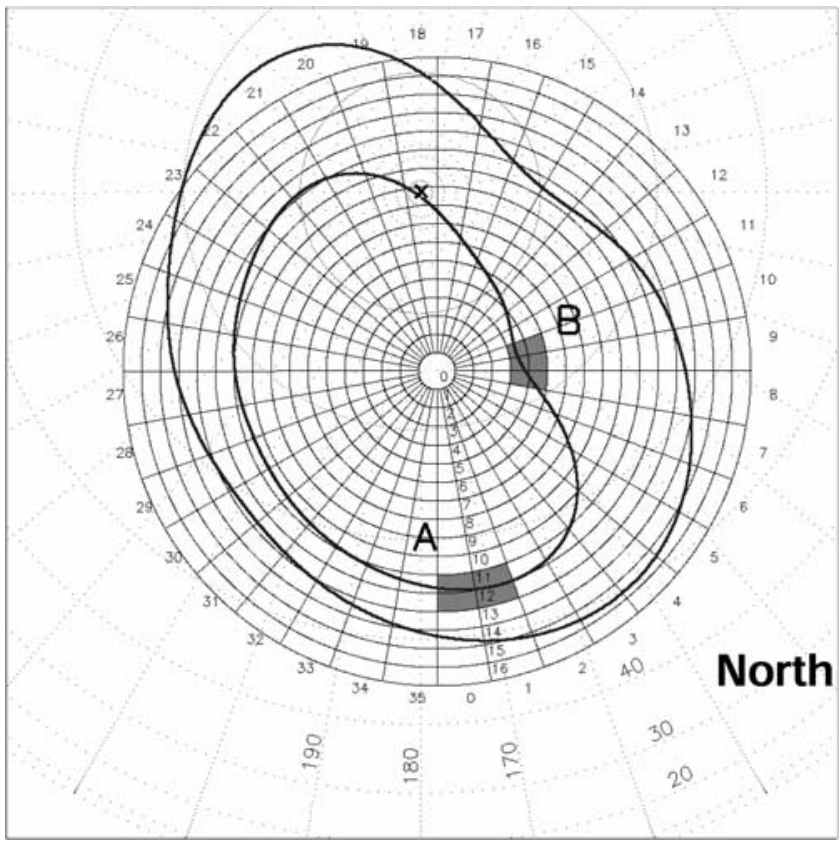

Figure 9. Segmentation of the northern auroral region in pseudo-magnetic coordinates used to estimate magnetic local times. The segmentation is realized on polar projected images using an off-axis polar mesh (solid line grid). The jovicentric pole is located with a cross mark while the center position of the mesh $\left(\lambda_{I I I}=175^{\circ}\right.$, latitude $\left.=75^{\circ}\right)$ roughly corresponds to the center of the reference ovals (plotted with thick solid lines). A $10^{\circ}$ spaced longitude-latitude grid (dotted lines) is overplotted. This arrangement allows one to pick up different auroral features along the ovals as well as inside the polar region and to analyze their variation as a function of CML or MLT. A correction for limb brightening with a cosine function is included. The shaded regions A and $\mathrm{B}$ are used to highlight the MLT modulation of the main oval brightness, as shown in Figure 10.

[29] The contraction is best seen in the $150-190^{\circ} \lambda_{I I I}$ region, though it is probably an effect of CML sampling and limb fitting and seems to vanish in the kink region. There is presently no clear physical interpretation for this contraction. It may be related to the compression of the dayside magnetosphere and is consistent with the day/night current pattern asymmetries that are expected and may be explained by changes in the radial outflow velocity as well [Bunce and Cowley, 2001a, 2001b]. According to Hill [2001], the distance at which corotation breaks down (in the centered dipole approximation) is inversely proportional to the outward mass transport rate. A doubling of the rate should produce an equatorward shift in the night side of the main oval of $\sim 1^{\circ}$. A latitude shift of $\sim 2^{\circ}$ would require a plausible factor of 4 larger mass transport rate at midnight than at noon (T. W. Hill, personal communication, 2002). However, as just indicated, these estimates are based on a dipole magnetic field approximation, and somewhat smaller latitudinal shifts are found when a more realistic current sheet model is employed [Nichols and Cowley, 2003].

[30] A further comparison of images taken at the same CML but on different days reveals that a second contrac- tion/expansion scheme of similar magnitude is superimposed on the motion described above. It can be discriminated from the first scheme because it takes place on a longer timescale (1 Earth day) and seems to affect the entire oval. During the observation campaign it appears that the main oval was smaller than the reference oval on 14 December 2000, 13 January 2001, and 20 January 2001. The most pronounced effect occurred on 13 January 2000 with a maximum deviation of almost $3^{\circ}$ at $\lambda_{I I I} \sim 190^{\circ}$. On the contrary, the main oval was slightly larger than the reference oval on 18 December 2000. Huddleston et al. [1998], Kivelson et al. [2001], and Khurana [2001] considered the solar wind influence on the Jovian magnetosphere. They all reach the conclusion that the Jovian magnetosphere is much more influenced by solar wind pressure than previously thought. Therefore it is expected that some contraction/expansion scheme, similar to that occurring for the Earth, may also apply to Jupiter, although the mechanism may not be the same. It will be seen in the last section that an interplanetary shock that hit Jupiter on 7 December 2000 was followed by a period of enhanced hectometric radio emissions. It suggests the possibility that the resulting compression/expansion phases of the magnetosphere might have continued until 14 December 2000.

[31] Although the overall structure of the kink is fixed in $\lambda_{I I I}$ (it appears to be the same at all CMLs and Galileo partly imaged it on the nightside), the brightness distribution along this main oval feature is usually found to slide clockwise along it, i.e., to lag corotation. A bright feature appearing in a series of nine consecutive images taken on 14 December 2000 has been used as a marker for measuring the degree of corotation of the emission in this region. Consecutive images covering $\sim 50^{\circ}$ of rotation show that this feature slid along the main oval. This motion has been mapped out to the magnetosphere with the VIP4 model and has been found to correspond to a $25^{\circ}$ rotation in the equatorial plane of Jupiter in $87 \mathrm{~min}$, that is, a subcorotation by a factor of 2 . According to Galileo data [Krupp et al., 2001], such a lag behind corotation is observed in the dusk plasma sheet in regions mapping from 15 to $55 \mathrm{R}_{J}$, that is, still in the middle magnetosphere.

\subsubsection{Changes in Brightness}

[32] To examine changes in brightness with MLT, the northern auroral region has been divided into $36 \times$ 16 segments as shown in Figure 9. The division is performed on polar projected images using an off-axis polar mesh. The center position of the mesh roughly corresponds to the center of the reference ovals and allows one to pick up different auroral features along the ovals as well as inside the main oval. This segmentation of the northern images obtained during the campaign permits one to measure separate local time variations of the brightness as Jupiter rotates. We also include a correction for limb brightening with a cosine function as a simple correction for the angle of each emission region to the line of sight consistent with optically thin emissions, as expected for the $\mathrm{H}_{2}$ and Doppler-broadened H Ly $\alpha$ emissions. The application was restricted to the auroral zones where this correction is valid (i.e., $20^{\circ}$ away from the limb). This method provides a simple way to compare the brightness of auroral features, presumably fixed in $\lambda_{I I I}$, as a function of time. More specifically, the brightness of any $\lambda_{I I I}$ segment can be traced 

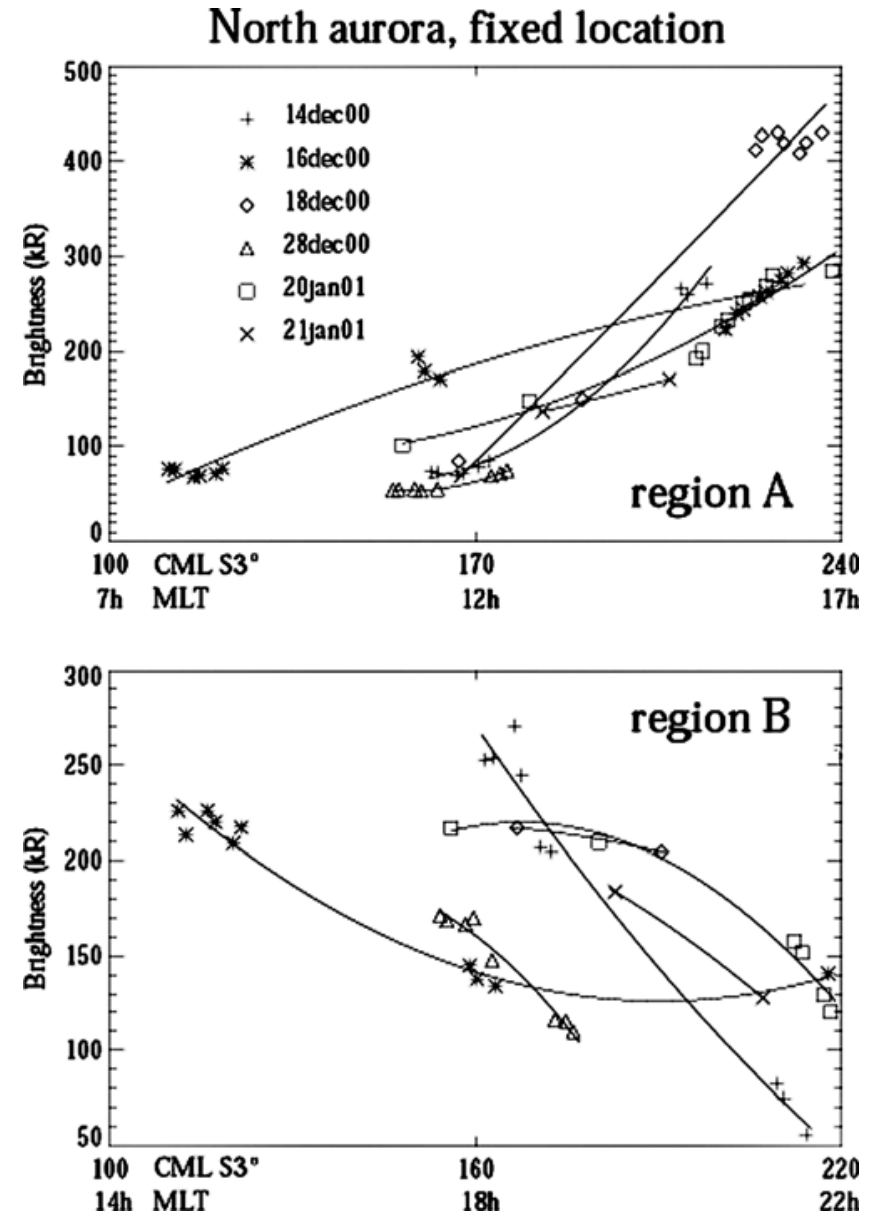

Figure 10. Illustration of the magnetic local time brightness variation observed in regions $\mathrm{A}$ and $\mathrm{B}$ of the main oval defined in Figure 9. Different observation days are represented with different symbols. The upper panel shows the brightness increase of region A when the CML increases from $100^{\circ}$ to $240^{\circ}$ (S3), that is when the MLT changes from 0700 to 1700 (derived from the VIP4 model, assuming a Jovian day divided in 24 "hours"). The lower panel shows the brightness decrease of region $\mathrm{B}$ for CML increasing from $100^{\circ}$ to $220^{\circ}$, corresponding to a MLT variation from 1400 to 2200 . The MLT trend is highlighted with a second order polynomial interpolation of the data points (solid lines).

among all the images for a given magnetic local time (that is, the local time at the magnetospheric region associated with an auroral sector) or at a given time as a function of the magnetic local time. The application of the method to the main auroral oval reveals a general trend in the modulation of the brightness with MLT: the brightness increases from noon to dusk, and then decreases again in the magnetic evening sector.

[33] This trend is illustrated in the upper and lower panels of Figure 10 which show a brightness increase from noon to dusk in a sector of the main oval centered near $\lambda_{I I I}$ longitude $170^{\circ}$ (shaded region $\mathrm{A}$ in Figure 9) and a brightness decrease from dusk to the premidnight sector in a sector around $150^{\circ}$ (shaded region B in Figure 9), respectively. The brightness in region $\mathrm{A}$ increases when the
CML changes from $120^{\circ}$ to $240^{\circ}$, that is (according to the VIP4 model), when the associated equatorial plasma region rotates from $\sim 0900$ MLT to $\sim 1700$ MLT (note that the MLT times given here conveniently assume a Jovian day divided in 24 "hours," with noon = 1200). On the other hand, the brightness in region B decreases when the CML increases from $120^{\circ}$ to $220^{\circ}$, that is, when the MLT increases from $\sim 1500$ to $\sim 2200$. A similar trend, though weaker, appears in other sectors of the main oval. Table 5 lists the brightness variation in a series of sectors along the main auroral oval. The 13 January 2001 does not appear in Table 5 and was not accounted for in Figure 10 because region $\mathrm{B}$ is not accessible in images with a CML $\geq 220^{\circ}$ and because on that day the aurora appeared atypical. As discussed in section 3.2 the bulk of the emission was considerably brighter on 13 January 2001. A more detailed analysis shows that each segment was affected by this brightening with emission features exceeding $1 \mathrm{MR}$ along the main oval versus an average value of $\sim 300 \mathrm{kR}$ for the other days. The brightness of the $\lambda_{I I I} 210^{\circ}-270^{\circ}$ sector of the main oval varied from $70 \mathrm{kR}$ (18 December 2000) to $300 \mathrm{kR}$ (20 January 2001) with localized peaks at $400 \mathrm{kR}$. No particular MLT trend was visible in this longitude range. The $190^{\circ}-210^{\circ}$ range usually does not show much activity with a brightness varying from 50 to $200 \mathrm{kR}$. A MLT variation emerges in the $180^{\circ}-190^{\circ}$ longitude sector where all data sets show a brightness increase (by a factor of $\sim 3$ ) when the CML increases from $200^{\circ}$. According to the VIP4 magnetic model, this sector, when observed at $\mathrm{CML}=200^{\circ}$, maps to a region of the magnetosphere at local noon. An even larger brightness increase (by a factor of $\sim 5$ ) occurs for the $160^{\circ}-180^{\circ}$ longitude sector (which includes "region $A$ " in the upper panel of Figure 10) when the CML increases from $120^{\circ}$. The brightness variation gradually decreases in the $140^{\circ}-160^{\circ}$ for $\mathrm{CML}$ varying from $100^{\circ}$ to $200^{\circ}$, that is when it rotates from dusk towards midnight, as shown for "region B" in the lower panel of Figure 10.

[34] The variation of auroral luminosity reported here appears to be consistent with the local time variation of the equatorial plasma velocity reported by Krupp et al. [2001] from analysis of energetic ion anisotropies. These data suggest the occurrence of rapid near-corotational flows in the dawn to noon sector, followed by slowing at dusk, and speeding up again in the premidnight sector. As explained above, according to the model by Cowley and Bunce [2001], the auroral intensity is expected to be regulated by the extent to which the equatorial plasma departs from rigid corotation with the planet, with the largest coupling currents and the most intense auroras being associated with the largest departures. The observations of Krupp et al. [2001] thus suggest auroras of modest intensity

Table 5. Brightness Variation in the Main Auroral Oval ${ }^{\mathrm{a}}$

\begin{tabular}{ccc}
\hline Longitude Range $^{\circ} \lambda_{I I I}$ & Brightness (kR) & Note \\
\hline $210-270$ & $70-300$ & localized peaks at $400 \mathrm{kR}$ \\
$190-210$ & $50-200$ & steady brightness \\
$180-190$ & $70-300$ & increase for CML $\geq 200^{\circ}$ \\
$160-180$ & $50-600$ & increase for CML $\geq 120^{\circ}$ \\
$140-160$ & $100-500$ & vanishing MLT trend \\
150 (poleward) & $100-300$ & decrease for CML $\geq 120^{\circ}$ \\
\hline
\end{tabular}

${ }^{\mathrm{a}}$ This table does not include data from 13 January 2001, during which the main oval was atypically bright. 
Multiple dawn arcs on 16 Dec. 2000

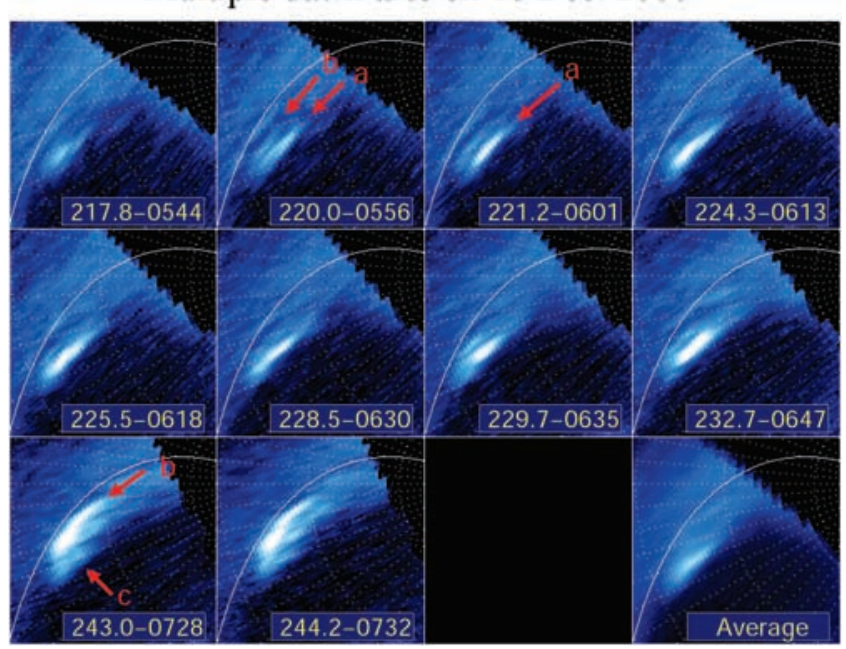

Figure 11. North polar projection of 10 consecutive images taken on 16 December 2000 showing the evolution of a multiple dawn arcs feature (arrows) in $\lambda_{I I I}$ coordinates relative to the main reference oval (solid line). The numbers give the CML, in $\lambda_{I I I}$ degrees, and the MLT of the feature, in hours (derived from the VIP4 model, assuming a Jovian day divided in 24 "hours"). A $5^{\circ}$ spaced grid is overplotted, the lowest parallel displayed is at $75^{\circ}$, and the horizontal dotted line is the $\lambda_{I I I} 270^{\circ}$ meridian. The last image (lower right panel) displays the average of the 10 images. The a, b, c arrows mark the individual features that form the MDA. At $\mathrm{CML}=221.2^{\circ}$, the brightness of arc a increases. At CML $=$ $243.0^{\circ}$, the $\mathrm{b}$ arc brightens and a new arc (c) appears.

in the dawn to noon sector where the plasma is nearcorotational, a brightening as the plasma slows in the postnoon and dusk sector, followed by a reduction once more as the plasma speeds up in the premidnight and midnight sectors. These expectations are thus compatible with the results shown here in Figure 10.

\subsection{Multiple Dawn Arcs (MDA)}

[35] Dawn storms, similar to the remarkable event observed for the first time in July 1993 with the FOC camera [Gérard et al., 1994b], have been occasionally detected since then in WFPC-2 images [Ballester et al., 1996; Clarke et al., 1998]. Dawn storms have been defined from earlier observations as occurring along the main oval, exceeding $1 \mathrm{MR}$ in brightness at the peak and remaining fixed in magnetic local time [Ballester et al., 1996; Clarke et al., 1998]. Such a bright event was detected during the campaign on 13 January 2001. On that day, the brightness of the main oval in the $220-270^{\circ} \lambda_{I I I}$ sector exceeded $1 \mathrm{MR}$ in two images taken $40 \mathrm{~min}$ apart. However, the whole oval appeared bright on that day, comparable to the brightness observed in the dawn sector. Recently, Cowley et al. [2003] have suggested that dawn storms may be associated with substorm-like magnetic reconnection events in Jupiter's magnetic tail, connected either with the solar wind-driven Dungey [1961] cycle or the rotation-driven cycle described by Vasyliūnas [1983].

[36] On 16 December 2000 a bright auroral emission developed at the poleward edge of the morning side main oval. We will show that this new kind of feature is different from the dawn storm described above in many regards: (1) it is in corotation with the planet, (2) it is located poleward of the main oval, and (3) it presents a complex morphology consisting of parallel arcs. Accordingly, we will refer to it as a "multiple dawn arcs" (MDA) feature. The development of the MDA feature was observed from the start of its development for a $\sim 100$-min period. After correction for limb brightening with a cosine function and subtraction of the background emission, the vertical brightness increased from $\sim 100 k R$ to $\sim 300 k R$ in 1.6 hours, i.e., generally fainter than the brightness usually observed in a dawn storm (a few MR). Figure 11 displays 10 images during which the feature was visible. The CML is displayed beside the magnetic local time (MLT) that maps the brightest spot in the emission to the equatorial plane with the VIP4 model. Various conclusions can be drawn from that figure. The brightest spot is located at a $\lambda_{I I I}$ longitude of $\sim 255^{\circ}$ and a latitude of $77^{\circ}$. This position remained constant over 1.6 hours, such that we can assert that the MDA were in corotation (within 10\%) with the planet. This corotation contrasts with the behavior of the dawn storms, which have been shown to lag corotation and remain fixed in MLT [Ballester et al., 1996]. The dawn storms have also been shown to appear consistently along the main oval [Clarke et al., 1998]. Comparison with the reference ovals shows that the MDA feature here is located poleward of the main oval and therefore maps farther into the magnetosphere.

[37] The spatial extent of the bright arc (feature $a$ in Figure 11) is $\sim 4000 \mathrm{~km}$ in the main oval direction by $\sim 500 \mathrm{~km}$ across it. This remained almost unchanged during the 1.6-hour observation time. It was previously assumed that a dawn storm could be seen as a single arc feature. The present STIS data set reveals a more subtle morphology of the MDA feature. Figure 11 shows that the emission consists of at least two narrow parallel arcs (arcs a and b). Arc a (poleward) remains brighter than $b$ during the whole observation. When mapped with the VIP4 model, it can be seen that arc a was already bright at 0544 MLT (assuming a Jovian day divided in 24 "hours," with noon $=1200$ ) then started to brighten at 0601 . Arc b, equatorward of a, started to brighten around 0728 MLT $(C M L=243.0)$. At the same time, a new faint arc (c) appeared poleward of b. It was recently proposed by Woch et al. [2002] from Galileo observations that the dawn storms may be driven by disruption of the rotational plasma flow due to explosive bursts of particles in the postmidnight tail region. In this picture, magnetic flux tubes heavily loaded with Iogenic plasma are stretched by centrifugal forces up to such a degree that spontaneous reconnection sets in, leading to acceleration of plasma and the release of plasmoids into interplanetary space (a process described by Carbary et al. [1976] and Vasyliūnas [1983]). The location of the MDA poleward of the main oval in the dawn sector suggests a distant magnetospheric origin, probably in the tail region. The corotational behavior of the MDA arcs described above and the timescale (hours) of the event also suggest that a disturbance, probably generated by an explosive burst, may have given rise to the observed UV feature. The proposed association of the new auroral features with plasmoid releases down the tail implies that these auroras are located on closed, near-corotating field lines downstream of the 
reconnection region which pinches off the plasmoid. This is inevitable because the plasmoid itself is essentially completely disconnected from the planet and thus itself cannot be related directly to auroral features. Near-rigid corotation of the closed field lines, postrelease, is not surprising after the plasmoid has carried away much of the plasma mass on the flux tubes. Auroras at the feet of the closed portions of the postrelease flux tubes could be related both to precipitation of plasma heated in the reconnection process that releases the plasmoid, and to the field-aligned currents that couple the changing angular momentum of the flux tubes between magnetosphere and ionosphere. Multiple structured emissions (Arcs a, b, and c in Figure 11) may then relate to individual plasmoid release episodes in the tail.

\subsection{Comparison With Galileo and Cassini Hectometric Emissions}

[38] Gurnett et al. [2002] report simultaneous observations using the Cassini and Galileo spacecraft of hectometric radio emissions and extreme UV (EUV) auroral emissions of Jupiter. They show that both of these emissions increased after the arrival at Jupiter of an interplanetary shock propagating outward from the Sun. When such a shock arrives at Jupiter, one would expect the magnetosphere to become strongly compressed. By analogy with the terrestrial case during geomagnetic storms, strong electric fields and therefore electron acceleration could be produced along the highlatitude auroral field lines, leading to a brightening of the auroral emissions. By contrast, Southwood and Kivelson [2001] and Cowley and Bunce [2001] have proposed that the main oval should decrease in brightness in response to increases in solar wind pressure at Jupiter. This follows from the expectation that when the magnetosphere is compressed, the angular velocities of the equatorial plasma will be enhanced, leading to reduced coupling currents and auroras, an expectation confirmed by the model calculations of Cowley and Bunce [2003a, 2003b]. If then the auroral emissions and associated radio phenomena are observed to be enhanced by shock-induced compressions of the magnetosphere, it must be due to a mechanism other than those discussed by these authors. Note, however, that there is no information on which of the three auroral emission regions (main oval, footprints, or polar regions) brightened at this time. One cannot therefore comment on the significance of the results reported by Gurnett et al. [2002] to the discussion about whether Jupiter's main oval becomes fainter when the solar wind pressure increases.

[39] Large variations have been reported in Jupiter's hectometric radio emission intensity during the period from 15 November 2000 to 14 January 2001. Three well-defined periods of enhanced hectometric radiation were evident, with the magnetometer and plasma measurements on Cassini showing that each of these events was preceded by an interplanetary shock. The second event started on 9 December 2000 and extended to 16 December 2000. Since the trapped continuum radiation appearing in Galileo's emission spectrogram (Figure 4 of Gurnett et al. [2002]) indicates that Galileo caught up the magnetopause on 13 December 2000 (doy 348), then it is likely that the compression had stopped at that time. Therefore the contraction of the main oval that occurred on 14 December 2000, mentioned in section 3.3.1, and the slight total emission increase (Figure 7) may correspond to the subsequent expansion phase of the magnetosphere. Accordingly, it may be suggested that the contraction of the main oval observed on that day is likely associated with an expansion of the magnetosphere rather than with a compression of the magnetosphere. However, it should be noted that a uniform compression of the magnetosphere alone will not change the position of the ionospheric feet of the field lines. To change the distribution of precipitation then requires the properties of the plasma to change on particular field lines.

[40] Gurnett et al. [2002] also observed a peak in the hectometric radiation intensity that occurred close to the time at which the maximum brightness of the EUV auoral emission was detected by the ultraviolet spectrograph on Cassini on 21 November 2000. This was near the time of maximum solar wind plasma density associated with an interplanetary shock that arrived at Jupiter on 18 November 2000. While there were no HST observations to correlate with the Cassini EUV observations in November 2000, the HST images did show a pronounced brightening and a contraction of the main oval on 13 January 2001. This behavior may be related to the solar wind density increase (one component of the solar wind dynamic pressure) observed by Cassini (W.S. Kurth, personal communication, 2002) on 13 January 2001. On that day the density was by far higher $\left(\sim 1 \mathrm{~cm}^{-3}\right)$ than on the other days of the campaign $\left(\leq 0.2 \mathrm{~cm}^{-3}\right)$. However, it should be noted that information on the solar wind was only available for a portion of the day in question. Further analysis of the Cassini and Galileo spacecraft [Kurth et al., 2002] suggest that on 10 January 2001 the jovian magnetosphere was compressed by increasing solar-wind dynamic pressure. However, there is no information on the size of the magnetosphere on 13 January 2001 so that it cannot be asserted whether the brighter and smaller main oval observed on that day relate to a compression or to an expansion phase of the magnetosphere.

\section{Summary}

[41] This paper reports the results of an imaging study of Jupiter's main auroral oval observed between December 2000 and January 2001. We have discussed the limitations inherent in the HST-STIS image processing and, more specifically, on the polar projection method that is affected by the pointing accuracy of HST-STIS.

[42] 1. A long term comparison of the images shows that the bulk of the auroral morphology is fixed in System III and remained stable over a 5-year period.

[43] 2. The 188 FUV images in the north and in the south allowed us to derive new reference ovals for the main oval, connecting the bright aligned auroral emission, and for the Io footprint oval.

[44] 3. A winding emission pattern in the $110-150^{\circ} \lambda_{I I I}$ region (the "kink") is always present in the two north ovals. Its bulk structure is both repeatable and in clear corotation. It is proposed that it stems from an internal magnetic anomaly that locally deforms the surface magnetic field. There is no clear evidence for a similar deformation at conjugate points in the south.

[45] 4. The main oval usually contributes to $\sim 75 \%$ of the the auroral brightness integrated over the pole. The total 
auroral brightness as a function of time does not show dramatic variation during the observation campaign, except for 13 January 2001 when the brightness of the entire main oval increased by a factor of two.

[46] 5. Analysis of the images as a function of CML reveals morphological variations of the main auroral oval. The main oval is shown to contract by $\sim 2^{\circ} \pm 0.8^{\circ}$ in latitude when the CML increases from 115 to $255^{\circ}$.

[47] 6. A secondary contraction, of the same order of magnitude, is found to take place on a longer timescale. It appears that the main oval was smaller than the reference oval on 14 December 2000, 13 January 2001, and 20 January 2001 , with the most pronounced effect occurring on 13 January 2001.

[48] 7. A bright auroral spot sliding along the main oval shows that while the kink pattern corotates with the planet, individual auroral features forming this structure lag corotation by a factor of two suggesting that the kink region maps to the middle magnetosphere, between 15 and $55 \mathrm{R}_{J}$.

[49] 8. The brightness of the main oval is shown to vary from 50 to $500 \mathrm{kR}$ apart from 13 January 2001 when the entire oval brightened up to more than $1 \mathrm{MR}$. The associated input power is on the order of 0.2 to $2 \times 10^{10} \mathrm{~W}$ per degree of arc in the thinnest parts of the oval and about three times more in the wider regions.

[50] 9. A statistical analysis of the main oval as a function of the CML reveals a modulation of the brightness which, for some sectors of the main oval, increases from noon to dusk and then decreases again in the magnetic evening.

[51] 10. A new auroral feature that we refere to as "multiple dawn arcs" has been observed during 1.6 hours. This feature is shown to be different from the dawn storms that have been observed in the past in many regards: (1) it is in corotation with the planet, (2) it is located poleward of the main oval, and (3) it presents a complex morphology, consisting of independent arcs.

[52] 11. Hectometric emissions observed simultaneously with Galileo and Cassini were shown to be related to three interplanetary shocks propagating outward from the Sun. It is suggested that the contraction of the main auroral oval observed in the HST images taken on 14 December 2000 may correspond to the expansion phase of the magnetosphere that followed the compression induced by the arrival of the second interplanetary shock. A brightening and a contraction of the main oval observed on 13 January 2001 may correspond to a time of increased solar wind dynamic pressure.

[53] Acknowledgments. The authors would like to thank Tom Hill for his useful comments on the contraction of the main oval. We are grateful to Jean-Claude Gérard for his thorough reading of this paper. The authors also thank the two referees for their valuable comments and suggestions. This work is based on observations with the NASA/ESA Hubble Space Telescope, obtained at the Space Telescope Science Institute (STScI), which is operated by AURA, Inc. for NASA under contract NAS5-26555. The research was supported by grant GO-08657.01-A from the Space Telescope Science Institute. DG is supported by the Belgian Fund for Scientific Research (FNRS) and SWHC by PPARC Senior Fellowship PPA/N/S/ 2000/00197.

[54] Arthur Richmond thanks the reviewers for their assistance in evaluating this paper.

\section{References}

Ballester, G. E., et al., Time-resolved observations of Jupiter's far-ultraviolet aurora, Science, 274, 409-412, 1996.
Broadfoot, A. L., B. R. Sandel, D. E. Shemansky, J. C. McConnell, G. R. Smith, J. B. Holberg, S. K. Atreya, T. M. Donahue, D. F. Strobel, and J. L. Bertaux, Overview of the Voyager ultraviolet spectrometry results through Jupiter encounter, J. Geophys. Res., 86, 8259-8284, 1981.

Bunce, E. J., and S. W. H. Cowley, Divergence of the equatorial current in the dawn sector of Jupiter's magnetosphere, Planet. Space Sci., 49, 1089-1113, 2001a.

Bunce, E. J., and S. W. H. Cowley, Local time asymmetry of the equatorial current sheet in Jupiter's magnetosphere, Planet. Space Sci., 49, $261-$ 274, 2001b.

Carbary, J. F., T. W. Hill, and A. J. Dessler, Planetary spin period acceleration of particles in the jovian magnetosphere, J. Geophys. Res., 81, 5189-5195, 1976.

Clarke, J. T., H. W. Moos, S. K. Atreya, and A. L. Lane, Observations from earth and variability of the polar aurora of Jupiter, Astrophys. J., 241, $179-183,1980$.

Clarke, J. T., et al., Hubble Space Telescope far-ultraviolet imaging of Jupiter during the impacts of Comet Shoemaker-Levy 9, Science, 267, 1302-1307, 1995.

Clarke, J. T., et al., Far-UV imaging of Jupiter's aurora with HST/WFPC 2, Science, 274, 404-409, 1996.

Clarke, J. T., et al., Hubble Space Telescope imaging of Jupiter's UV aurora during the Galileo orbiter mission, J. Geophys. Res, 103, 20,217-20,236, 1998 .

Clarke, J. T., et al., Ultraviolet auroral emissions from the magnetic footprints of Io, Ganymede, and Europa on Jupiter, Nature, 415, 997-1000, 2002a.

Clarke, J. T., D. Grodent, J. E. P. Connerney, The distorted shape of Jupiter's northern auroral oval-A possible magnetic anomaly, paper presented at the 34th Annual Meeting, Div. for Planet. Sci., Birmingham, Ala., October, 2002b.

Connerney, J. E. P., M. H. Acuña, N. F. Ness, and T. Satoh, New models of Jupiter's magnetic field constrained by the Io flux tube footprint, J. Geophys. Res., 103, 11,929-11,939, 1998.

Cowley, S. W. H., and E. J. Bunce, Origin of the main auroral oval in Jupiter's coupled magnetosphere-ionosphere system, Planet. Space Sci., 49, 1067-1088, 2001.

Cowley, S. W. H., and E. J. Bunce, Modulation of jovian middle magnetosphere currents and auroral precipitation by solar wind-induced compressions and expansions of the magnetosphere: Initial conditions and steady state, Planet. Space Sci., 51, 31-56, 2003a.

Cowley, S. W. H., and E. J. Bunce, Modulation of Jupiter's main auroral oval emissions by solar wind-induced expansions and compressions of the magnetosphere, Planet. Space Sci., 51, 57-79, 2003b.

Cowley, S. W. H., J. D. Nichols, and E. J. Bunce, Distributions of current and auroral precipitation in Jupiter's middle magnetosphere computed from steady-state Hill-Pontius angular velocity profiles: Solutions for current sheet and dipole magnetic field models, Planet. Space Sci., 50, 717-734, 2002

Cowley, S. W. H., E. J. Bunce, T. S. Stallard, and S. Miller, Jupiter's polar ionospheric flows: Theoretical interpretation, Geophys. Res. Lett., 30(5), 1220, doi:10.1029/2002GL016030, 2003.

Dessler, A. J., and V. M. Vasyliūnas, The magnetic anomaly model of the Jovian magnetosphere: Predictions for Voyager, Geophys. Res. Lett., 6, $37-40,1979$.

Dungey, J. W., Interplanetary field and the auroral zones, Phys. Rev. Lett., 6, 47-49, 1961.

Gérard, J.-C., V. Dols, R. Prangé, and F. Paresce, The morphology of the north Jovian ultraviolet aurora observed with the Hubble Space Telescope, Planet. Space. Sci., 42, 905-917, 1994a.

Gérard, J.-C., D. Grodent, R. Prangé, J. H. Waite, G. R. Gladstone, V. Dols F. Paresce, A. Storrs, L. Ben Jaffel, and K. A. Franke, A remarkable auroral event on Jupiter observed in the ultraviolet with the Hubble Space Telescope, Science, 266, 1675-1678, 1994b.

Gérard, J.-C., J. Gustin, D. Grodent, P. Delamere, and J. T. Clarke, Excitation of the FUV Io tail on Jupiter: Characterization of the electron precipitation, J. Geophys. Res., 107(A11), 1394, doi:10.1029/2002JA009410, 2002.

Gladstone, G. R., et al., A pulsating auroral X-ray hot spot on Jupiter, Nature, 415, 1000-1003, 2002.

Grodent, D., G. R. Gladstone, J.-C. Gérard, V. Dols, and J. H. Waite, Simulation of the norphology of the Jovian UV north aurora observed with the Hubble Space Telescope, Icarus, 128, 306-321, 1997.

Grodent, D., J. H. Waite Jr., and J.-C. Gérard, A self-consistent model of the Jovian auroral thermal structure, J. Geophys. Res., 106, 12,933-12,952, 2001a.

Grodent, D., J. Gustin, J.-C. Gérard, J. H. Waite, and J. T. Clarke, High color ratio and high temperature in Jupiter's auroral atmosphere, paper presented at the 33rd Annual Meeting, Div. for Planet. Sci., New Orleans, La., 2001b. 
Grodent, D., J. T. Clarke, J. H. Waite Jr., S. W. H. Cowley, J.-C. Gérard, and J. Kim, Jupiter's polar auroral emissions, J. Geophys. Res., 108, doi:10.1029/2003JA010017, in press, 2003.

Gurnett, D. A., et al., Control of Jupiter's radio emission and aurorae by the solar wind, Nature, 415, 985-987, 2002.

Gustin, J., D. Grodent, J.-C. Gérard, and J. T. Clarke, Spatially resolved far ultraviolet spectroscopy of the Jovian aurora, Icarus, 157, 91-103, 2002.

Harris, W., J. T. Clarke, M. A. McGrath, and G. E. Ballester, Analysis of Jovian auroral H Ly- $\alpha$ emission (1981-1991), Icarus, 124, 350-365, 1996.

Hill, T. W., Corotation lag in Jupiter's magnetosphere: Comparison of observations and theory, Science, 207, 301-302, 1980.

Hill, T. W., The Jovian auroral oval, J. Geophys. Res., 106, 8101-8107, 2001.

Hill, T. W., and V. M. Vasyliūnas, Jovian auroral signature of Io's corotational wake, J. Geophys. Res., 107(A12), 1464, doi:10.1029/2002JA009514, 2002

Huddleston, D. E., C. T. Russell, M. G. Kivelson, K. K. Khurana, and L. Bennett, Location and shape of the Jovian magnetopause and bow shock, J. Geophys. Res., 103, 20,075-20,082, 1998.

Khurana, K. K., Influence of solar wind on Jupiter's magnetosphere deduced from currents in the equatorial plane, J. Geophys. Res., 106, 25,999-26,016, 2001.

Kivelson, M. G., D. J. Southwood, K. K. Khurana, C. T. Russell, and R. J. Walker, Jupiter's dynamic magnetosphere, paper presented at Jupiter: Planet, Satellites and Magnetosphere, Lab. for Atmos. and Space Phys., Univ. of Colo., Boulder, Colo., 25-30 June 2001

Krupp, N., A. Lagg, S. Livi, B. Wilken, J. Woch, E. C. Roelof, and D. J. Williams, Global flows of energetic ions in Jupiter's equatorial plane: First-order approximation, J. Geophys. Res., 106, 26,017-26,032, 2001.

Krupp, N., et al., Leakage of energetic particles from Jupiter's dusk magnetosphere: Dual spacecraft observations, Geophys. Res. Lett., 29(15), 1736, doi:10.1029/2001GL014290, 2002.

Kurth, W. S., et al., The dusk flank of Jupiter's magnetosphere, Nature, 415 , 991-994, 2002.

Landsman, W., Characteristics of the FUV-MAMA dark rate, STScI Rep. Space Telescope Sci. Inst., Baltimore, Md., 1998. (Available at http:// hires.gsfc.nasa.gov/stis/postcal/quick_reports/r057/r057.html).

Livengood, T. A., H. W. Moos, G. E. Ballester, and R. Prangé, Jovian ultraviolet auroral activity, 1981-1991, Icarus, 97, 26-45, 1992.

Nichols, J. D., and S. W. H. Cowley, Magnetosphere-ionosphere coupling curents in Jupiter's middle magnetosphere: Dependence on the effective ionospheric Pedersen conductivity and iogenic plasma mass outflow rate, Ann. Geophys., 21, 1419-1441, 2003.
Pallier, L., and R. Prangé, More about the structure of the high latitude Jovian aurorae, Planet. Space Sci., 49, 1159-1173, 2001.

Prangé, R., D. Rego, L. Pallier, J. E. P. Connerney, P. Zarka, and J. Queinnec, Detailed study of FUV Jovian auroral features with the post-COSTAR HST faint object camera, J. Geophys. Res., 103, 20,195-20,215, 1998.

Sandel, B. R., et al., Extreme ultraviolet observations from Voyager 2 encounter with Jupiter, Science, 206, 962-966, 1979.

Sands, M. R., and R. L. McNutt, Plasma bulk flow in Jupiter's dayside middle magnetosphere, J. Geophys. Res., 93, 8502-8518, 1988.

Skinner, T. E., S. T. Durrance, P. D. Feldman, and H. W. Moos, IUE observations of longitudinal and temporal variations in the Jovian auroral emission, Astrophys. J., 278, 441-448, 1984.

Southwood, D. J., and M. G. Kivelson, A new perspective concerning the influence of the solar wind on the Jovian magnetosphere, J. Geophys. Res., 106, 6123-6130, 2001.

Stallard, T. S., S. Miller, G. Millward, and R. D. Joseph, On the dynamics of the jovian ionosphere and thermosphere I. The measurement of ion winds, Icarus, 154, 475-491, 2001.

Vasavada, A. R., A. H. Bouchez, A. P. Ingersoll, B. Little, C. D. Anger, and the Galileo SSI Team, Jupiter's visible aurora and Io footprint, J. Geophys. Res., 104, 27,133-27,142, 1999.

Vasyliūnas, V. M., Plasma distribution and flow, in Physics of the Jovian Magnetosphere, edited by A. J. Dessler, p. 395, Cambridge Univ. Press, New York, 1983.

Waite, J. H., Jr., et al., An auroral flare at Jupiter, Nature, 410, 787-789, 2001.

Walsh, J. R., P. Goudfrooij, and E. Malumuth, STIS geometric distortion SMOV3A tests for CCD, NUV-MAMA, and FUV-MAMA, Space Telescope Sci. Instrum. Rep. 2001-02, 2001.

Woch, J., N. Krupp, and A. Lagg, Particle bursts in the Jovian magnetosphere: Evidence for a near-Jupiter neutral line, Geophys. Res. Lett., 29(7), 1138, doi:10.1029/2001GL014080, 2002.

J. T. Clarke and J. Kim, CSP, Boston University, 725 Commonwealth Avenue, Boston, MA 02215, USA. (jclarke@bu.edu; juwhan@bu.edu)

S. W. H. Cowley, Department of Physics and Astronomy, Leicester University, Leicester, LE1 7RH, UK. (swhcl@ion.le.ac.uk)

D. Grodent, Institut d'Astrophysique et de Géophysique (B5c), Université de Liège, Allée du 6 août, 17, 4000 LIEGE, Belgium. (d.grodent@ ulg.ac.be)

J. H. Waite Jr., Space Physics Research Laboratory, University of Michigan, 2436B SRB, Ann Arbor, MI 48109-2143, USA. (hunterw@ umich.edu) 